\title{
Liame
}

Histoire et histoire de l'art des époques moderne et contemporaine de l'Europe méditerranéenne et de ses périphéries

25 | 2012

Les réseaux négociants dans la France méridionale (XVIII ${ }^{\mathrm{e}}$-XIX ${ }^{\mathrm{e}}$ siècles)

\section{Maisons de commerce bas-languedociennes et réseaux négociants méridionaux : l'exemple des Balguerie et des Fraissinet (XVIII ${ }^{\mathrm{e}}$-XIX ${ }^{\mathrm{e}}$ siècles)}

\section{Lionel Dumond}

\section{OpenEdition}

Journals

Édition électronique

URL : http://journals.openedition.org/liame/282

DOI : 10.4000/liame.282

ISSN : 2264-623X

Éditeur

CRISES - Centre de Recherches Interdisciplinaires en Sciences Humaines et Sociales de Montpellier

\section{Référence électronique}

Lionel Dumond, «Maisons de commerce bas-languedociennes et réseaux négociants méridionaux: l'exemple des Balguerie et des Fraissinet (XVIII-XIX siècles) », Liame [En ligne], 25 | 2012, mis en ligne le 05 décembre 2012, consulté le 02 mai 2019. URL : http://journals.openedition.org/liame/282 ; DOI $10.4000 /$ liame.282

Ce document a été généré automatiquement le 2 mai 2019.

\section{cc) (†) $\ominus$}

Les contenus de Liame sont mis à disposition selon les termes de la Licence Creative Commons Attribution - Pas d'Utilisation Commerciale - Pas de Modification 4.0 International. 


\title{
Maisons de commerce bas- languedociennes et réseaux négociants méridionaux: l'exemple des Balguerie et des Fraissinet (XVIII $-\mathrm{XIX} \mathrm{X}^{e}$ siècles)
}

\author{
Lionel Dumond
}

1 Figures emblématiques des dynamismes commerciaux phocéen et bordelais des $\mathrm{XVIII}^{\mathrm{e}}$ et XIX ${ }^{e}$ siècles, les familles Fraissinet et Balguerie constituent des exemples de choix pour l'étude du négoce méridional ${ }^{1}$. Jean Fraissinet et Jean-Étienne Balguerie junior étaient au début de la Révolution leurs représentants les plus en vue de Marseille et de Bordeaux. Ils avaient aussi pour point commun de partager la même origine montpelliéraine ${ }^{2}$. Ce faisant, ils offrent l'opportunité d'associer des espaces généralement envisagés de façon distincte, considérés ici à partir des maisons de commerce bas-languedociennes dont ils sont issus ${ }^{3}$. Renversant la perspective traditionnelle, construite depuis les deux ports mondiaux des midis de la France, ils offrent un point d'entrée original à l'analyse des réseaux négociants méridionaux. Ils questionnent en particulier la morphologie et les dynamiques des relations entretenues par le négoce de Montpellier-Sète en interrogeant les espaces sociaux sur lesquels elles reposent ${ }^{4}$. Ce faisant, ils permettent de combiner la distribution géographique de leurs réseaux avec des données longitudinales sur leurs trajectoires dans une approche désormais classique des réseaux sociaux ${ }^{5}$.

Inédite, une telle étude se heurte à l'absence des archives de leurs entreprises d'origine, une difficulté méthodologique responsable de leur faible empreinte historiographique qui fait qu'en dehors de Fernand Braudel évoquant la firme Fraissinet de Sète dans son rapport de maison-mère de Fraissinet fils d'Amsterdam, il n'en a presque jamais été fait mention ${ }^{6}$. L'obstacle documentaire peut cependant être surmonté par une investigation tous azimuts ${ }^{7}$. Les sources issues des archives départementales de l'Hérault et des Bouches-du-Rhône et, en particulier, de leurs fonds judiciaires, de leurs minutes notariales et de leurs registres d'état-civils, complétées par les informations des quartiers 
maritimes d'Agde et de Sète conservées à Toulon ainsi que par le fonds Fraissinet déposé aux archives municipales de Marseille livrent une documentation d'une richesse surprenante qui autorise l'esquisse des cheminements languedociens des Fraissinet et des Balguerie ${ }^{8}$.

Point de départ de cette étude, leurs maisons de commerce du milieu du XVIII ${ }^{\mathrm{e}}$ siècle présentent l'ensemble des caractéristiques propres aux grandes firmes locales adonnées au trafic des alcools (1). Leur proximité d'organisation et d'activité n'est pas contradictoire avec leur inscription dans deux trajectoires familiales dissemblables qui ont en commun leur appartenance au protestantisme et leurs liens avec le refuge amstellodamois (2). Ensemble, ces éléments donnent aux réseaux sur lesquels elles s'appuient des traits fréquemment voisins, définissant une sorte de modèle de réseau négociant centré sur le Bas-Languedoc (3).

\section{Deux grandes maisons de commerce montpelliéraines}

En 1753, les maisons de commerce Fraissinet et Balguerie appartiennent à la douzaine de firmes montpelliéraines que la Chambre de commerce de Montpellier cite pour leur domination du commerce sétois9. Fondées sur ces bases économiques similaires, elles présentent des formes d'organisation et d'activité relativement proches ${ }^{10}$.

\section{Des organisations bipolaires}

5 Les deux entreprises se caractérisent par une même organisation matérielle : leur double implantation à Montpellier et à Sète les font bénéficier de tous avantages qu'offrent la capitale du Bas-Languedoc et son port. Et leur organisation bipolaire se retrouve et se répercute dans leurs architectures sociales associant généralement deux individus.

Montpellier n'offre pas seulement des facilités qui relèvent de ses fonctions de commandement dans les ordres administratifs, judiciaires et économiques. La ville constitue aussi un environnement économique extrêmement dynamique. Elle soutient une intense activité financière qui fait dire au professeur Dermigny qu'elle constitue dans ce domaine la plus petite des grandes places internationales. C'est par elle que passe une très grande part des transactions régionales. Elle tire de son arrière-pays un large éventail de marchandises et elle fabrique elle-même quantité de produits variés. Entre autres spécialités, son verdet jouit d'une réputation européenne.

7 Vers 1750, l'activité d'Antoine Fraissinet, père du Jean Fraissinet cité plus haut, s'y déploie depuis un enclos limitrophe de l'enceinte de la ville, situé près de la porte du Peyrou, une propriété familiale acquise au siècle précédent à partir de laquelle travaillent également ses frères ${ }^{11}$. Quant à Jean Balguerie, père de Balguerie junior, il est d'abord présent dans la paroisse Saint-Denis. Puis il y multiplie les installations, avec la création vers 1754 d'une fabrique de savon et la construction cinq ans plus tard d'un "grand magasin $»^{12}$.

8 Les établissements portuaires des maisons de commerce montpelliéraines jouent un rôle essentiellement logistique. Chargés d'organiser les chargements des bâtiments de mer, ils comportent à la fois un comptoir, destiné à la tenue des livres et à la réception des capitaines, et des entrepôts ouverts sur le quai du canal d'où s'effectue l'embarquement de leurs marchandises. Ils emploient deux types de personnels spécialisés avec, d'une 
part, des commis fréquemment originaires du nord de l'Europe, recrutés pour leur capacité à communiquer avec les capitaines étrangers en escale, et, d'autre part, un personnel de cave placé sous l'autorité d'un maître de chai. Chargé de soigner les vins et les alcools placés sous sa garde, ce dernier ne limite pas son activité à veiller à leur bonne conservation ; il s'occupe aussi de toutes sortes de manipulation, -on parle localement de triturations-, destinées à les bonifier.

Au début des années 1750, les Fraissinet possèdent dans le port deux maisons donnant à la fois sur la Grand rue et sur le quai du canal. Le développement de leurs bienfonds suit une active politique de promotion immobilière qui fait qu'ils y détiennent en 1763 trois maisons et un grand magasin qu'ils ont fait construire sur la Rive neuve, de l'autre côté du canal ${ }^{13}$. Ces immobilisations constituent un capital facilement mobilisable, notamment utilisé pour dépasser leur faillite de cette année-là. Ce sont aussi de bonnes affaires en raison de l'élévation continue des prix de l'immobilier sétois ${ }^{14}$. En 1778, leur magasin de la rive neuve s'est ainsi valorisé de $60 \%$ en une quinzaine d'années, à $100.000 \mathrm{ft}$ contre 60.000 en $1763^{15}$. Il complète alors des installations qui comprennent à Sète deux autres magasins situés du côté de la ville, dont l'un sert de comptoir et l'autre, dénommé "magasin du dehors ", possède une fabrique d'eau-de-vie, et, en dehors de Sète, un magasin établi sur le port d'étang de Marseillan ${ }^{16}$.

En comparaison, l'établissement sétois de la maison Balguerie fait pâle figure. Il relève jusqu'en 1755 de Louis Rogé, l'associé de Jean Balguerie. Puis, après la séparation de Rogé \& Balguerie, Balguerie l'établit maison « veuve Péridier ». Là, il dispose d'un immeuble de deux étages avec comptoir, magasin et grenier, et d'un plus grand magasin adjacent, le tout donnant sur le quai du canal, du côté de la rive neuve ${ }^{17}$.

11 Bipolaires, les firmes Fraissinet et Balguerie réunissent fréquemment au moins deux individus. La nature de leurs activités les pousse continuellement à s'attacher les services de négociants originaires du Nord.

12 Jusqu'en 1760, les Fraissinet assurent la gestion de leur maison de commerce avec plusieurs membres de leur famille (voir figure $n^{\circ} 1$ ). Le fondateur de leur négoce baslanguedocien de liquides, Isaac Fraissinet, a pris deux fils Isaac et Antoine comme associés dès les années 1720 . Ceux-ci ont ensuite poursuivi l'entreprise après son décès ${ }^{18}$. Antoine était chargé des affaires sétoises. Il résida dans le port tout au long de sa vie professionnelle, sans cesser pour autant d'être considéré comme un négociant montpelliérain ${ }^{19}$. En 1749, le décès de son frère le laissa seul à la tête de la firme. Mais pour peu de temps, car il y fit entrer son fils Marc dès sa majorité, en 1753. Après sa propre disparition en 1760, Marc continua l'œuvre familiale depuis Montpellier. Il changea la raison sociale de l'entreprise pour celle de Marc Fraissinet \& C $C^{\text {ie }}$ à l'issue de son arrêt de 1763 , choisissant au même moment de s'établir à Sète ${ }^{20}$. L'importance cruciale du port dans l'activité des firmes montpelliéraine s'était déjà manifestée, deux ans plus tôt, avec l'installation de Balguerie à Sète ${ }^{21}$.

13 Dans sa direction d'entreprise, Marc Fraissinet était secondé par des commis nordiques dont on ignore précisément le statut. Il collabora successivement avec le brêmois André Piel et avec le natif de Stettin Jean-Louis Engelke. Piel arriva en Bas-Languedoc en 1766 après avoir été le commis du commissionnaire bordelais Joseph Arnaud, beau-frère de Marc Fraissinet ${ }^{22}$. Dans le port girondin, il a pu améliorer sa connaissance des pratiques du commerce des alcools ${ }^{23}$. Il crée ensuite sa propre maison sous la raison Piel \& Ricard. À partir de 1773, il est remplacé auprès de Marc Fraissinet par Engelke, un ancien commis du négociant sétois d'origine helvétique Jean Counclerc qui vient de devenir, par son 
mariage avec Marguerite Boischon un parent de Marc Fraissinet. Engelke continue après 1778 les affaires de l'ancienne maison de commerce Fraissinet.

On a déjà vu que Jean Balguerie travaille jusqu'en 1755 avec Louis Rogé. Son associé était auparavant celui du négociant néerlandais Antoine Verborg parti s'établir à Hambourg. Rogé et Balguerie ont des commis d'origine allemande: le premier travaille avec le hambourgeois Jean Flickwier dont il fait son gendre en 1751 ; le second avec ChrétienAbraham Seyffert qui, originaire de Leipzig, est le parrain de l'un de ses enfants en $1753^{24}$. Comme Engelke, Seyffert a séjourné à Bordeaux avant de rejoindre le Bas-Languedoc et, comme lui, il est amené à poursuivre les affaires de son associé après leur faillite et le décès de Jean Balguerie en $1764^{25}$. Il prolonge son commerce pendant plus de quarante ans ${ }^{26}$.

Figure $\mathrm{n}^{\circ} 1$ : les maisons Fraissinet et Balguerie de Montpellier-Sète

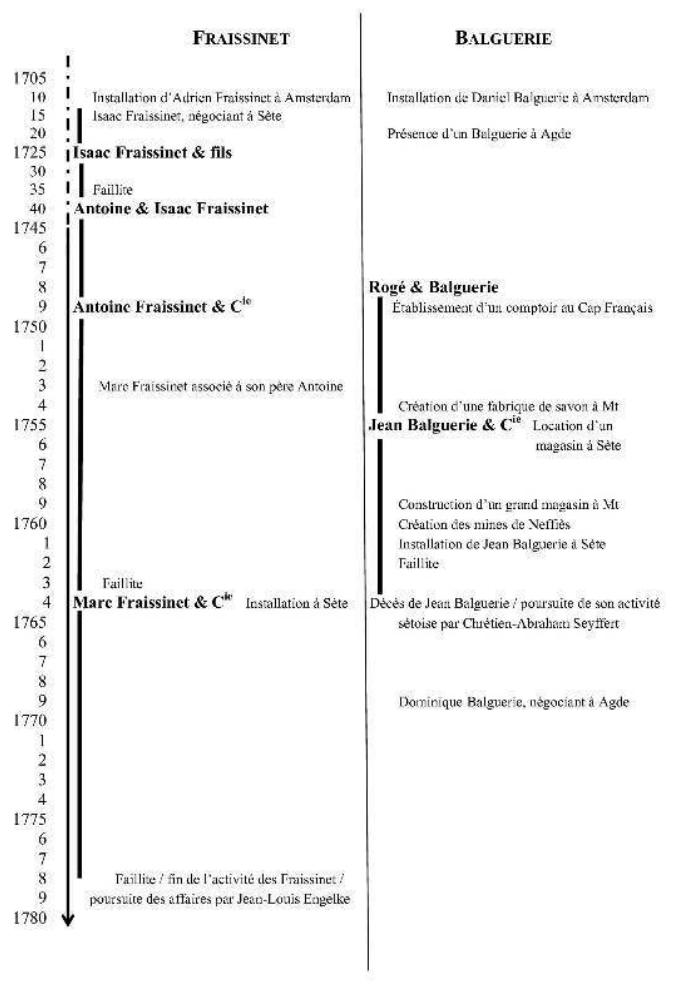

\section{Activités et conjonctures}

15 Adonnées aux mêmes trafics d'alcools, les maisons Fraissinet et Balguerie subissent les mêmes aléas conjoncturels qui les conduisent à s'arrêter avant d'obtenir un concordat de leurs créanciers leur permettant de prolonger leur activité. Elles présentent cependant quelques différences d'orientations commerciales, qui se traduisent dans la destination comme dans la nature de leurs affaires. Car leur commerce de liquides n'est pas exclusif d'autres d'opérations: il s'accompagne d'incursions dans d'autres secteurs d'activités, depuis l'armement de bâtiments de mer jusqu'à la production d'eaux-de-vie, de savons, voire, dans le cas de Balguerie, l'exploitation minière.

Leurs envois d'alcools vers le Nord s'effectuent selon des modalités variées. Ils se font le plus souvent à la commission, c'est-à-dire en réponse aux commandes de leurs 
correspondants ${ }^{27}$. Mais ils peuvent aussi s'effectuer pour leur propre compte, leurs marchandises étant alors placées en consignation chez un commerçant étranger. Dans ce cas de figure, ils partagent fréquemment le risque commercial avec leurs entrepositaires et ils compensent la modestie de leurs engagements par leur multiplicitén ${ }^{28}$.

La même prudence commande la dispersion de leurs envois sur de nombreux bâtiments qui limite les conséquences d'une éventuelle fortune de mer. Cette pratique se traduit localement par le grand nombre de maisons impliquées dans une même cargaison. En novembre 1754, le Jean-Jean, commandé par le capitaine Jean de Grrot, charge-t-il ainsi pour Amsterdam des produits de la plupart des grandes maisons montpelliéraines parmi lesquels on compte ceux d'Antoine Fraissinet \& fils et ceux de Rogé \& Balguerie.

Leur commerce s'étend à une gamme de liquides assez large pour justifier l'étendue de leurs entrepôts. Plusieurs inventaires de magasins sétois sont éloquents à cet égard. Ils montrent qu'à chaque catégorie d'alcool, -eaux de vie, muscats, vins du midi et vins étrangers-, correspond un mode de conditionnement préférentiel, en barriques, tonneaux ou bouteilles. Et que chacune de ces catégories se diversifie en bon nombre de types particuliers selon des critères qui lui sont propres, les eaux-de-vie en fonction de leur degré alcoolique, les plus courantes étant les «trois-six», les muscats selon leur couleur (blanc ou rouge) et leur provenance (Béziers, Frontignan, Lunel, etc.) ${ }^{29}$. Les vins régionaux donnent lieu à des appellations qui renvoient à leur âge (vins nouveaux, vins vieux), à leur qualité (première ou seconde qualité, vins de chaudière destinés à la distillation), à leur couleur (blanc ou rouge) ou encore à leurs zones de production désignées par ensembles géographiques (Roussillon, Rivesaltes, Rhône, etc.) ou par localités (Poussan, Marseillan, Saint-Georges, etc.). Les vins étrangers, enfin, ibériques ou italiens, sont décrits par leur provenance (Malaga, Porto, vin d'Alicante, vin de Calabre, etc.). Encore faut-il remarquer que ces classements, simples et objectifs en apparence, recouvrent en réalité des produits qui peuvent être falsifiés. Car les alcools sétois sont loin de toujours être ce qu'ils prétendent, l'art du maître de chai consistant non seulement à renforcer le caractère des vins les plus faibles, mais aussi à reproduire artificiellement certains breuvages d'appellations réputées.

Quoi qu'il en soit de ces pratiques, elles ne participent probablement que marginalement dans l'évolution extrêmement favorable du commerce des liquides bas-languedocien. En effet, le XVIII ${ }^{e}$ siècle voit la transformation de la demande du Nord en parfaite adéquation avec l'évolution structurelle de l'offre régionale. La production locale se plie aux tendances successives du marché. D'abord centrée sur les blancs ou picardans à la fin du $\mathrm{xVII}^{\mathrm{e}}$ siècle, elle s'attache durant le premier tiers du xvIII ${ }^{\mathrm{e}}$ siècle à étancher la soif d'eauxde-vie du Nord de l'Europe et particulièrement des maisons de commerce amstellodamoises. Pour cela, elle développe ses fabrications de vins de chaudière, véritable stimulant de nouvelles formes de développement de son vignoble. Puis, vers le milieu du siècle, l'accroissement de la consommation des vins rouges aux Provinces-Unies offre un puissant relai de croissance de cet essor. Il a l'intérêt de correspondre aux productions pour lesquelles cette viticulture est la plus naturellement douée ${ }^{30}$. Ce changement, qui confère aussi aux vignobles du Midi un avantage certain sur ceux situés plus au nord, donne au négoce sétois une nouvelle impulsion qui profite notamment aux firmes qui nous intéressent ici ${ }^{31}$.

Leurs envois de ses liquides s'accompagnent toujours d'un certain nombre d'articles de complément. Le chargement effectué en 1748 par Rogé \& Balguerie sur le vaisseau suédois La Fortuné à destination de Lübeck, fournit un exemple de cette variété car il comprend, 
en dehors des vins, des eaux-de-vie et des muscats, une série de caisses contenant des produits issus de l'agriculture, de la pêche et de l'industrie locale. On y dénombre ainsi deux caisses d'olives, trois caisses de parfum appelé eau de la reine de Hongrie, deux caisses de liqueurs, une caisse contenant dix barils d'anchois, un ballot de six pains de verdet et quatre balles d'amandes en coque ${ }^{32}$. D'autres envois s'accompagneraient aussi d'huiles d'olives, de liège ou de cuirs ${ }^{33}$. C'est assez dire que ces cargaisons de complément sollicitent tout l'éventail des productions méditerranéennes et contribuent à la notoriété de certaines spécialités industrielles locales, notamment dans les secteurs de la droguerie (parfums, verdet) et de la mégisserie.

21 L'aire géographique des activités des firmes Fraissinet et Balguerie ne se limite pas à l'Europe septentrionale. Elle comprend aussi notamment le bassin occidental de la Méditerranée dans lequel elles trafiquent d'autres types de produits. Là, sur d'assez courtes distances, elles s'occupent notamment de céréales, de bois, de cercles de futailles, de laines et même de draperii ${ }^{34}$. Commercialement, ce second réseau reste cependant d'une importance secondaire, comme en témoigne la faible place qu'il occupe dans leurs bilans. Celui de Marc Fraissinet établi en 1763 dessine bien cette hiérarchie : le commerce du Nord y correspond à toutes ses principales destinations. Derrière la Hollande, où se rencontre son principal créancier, se trouvent Hambourg et Saint-Pétersbourg. L'Espagne (Malaga, Cadix, Madrid, Barcelone), l'Italie (Gène), le Ponant (Rouen Dunkerque), la Méditerranée orientale (Le Caire), le Midi de la France (Marseille, Toulouse, Bordeaux), et enfin Genève, ne viennent que bien plus loin pour le montant de leurs engagements ${ }^{35}$. La même remarque vaudrait certainement pour Jean Balguerie dont le plus important créancier est en 1762 une firme d'Amsterdam ${ }^{36}$.

Hors du cadre européen, les firmes Balguerie et Fraissinet s'adonnent, apparemment de façon plus sporadique, au commerce des îles. Jean Balguerie charge ainsi pour les colonies françaises d'Amérique à la fin des années 1740. Il tente même d'organiser un comptoir au Cap français (Saint-Domingue) au début de la décennie suivante ${ }^{37}$. C'est un échec même si le lieu correspond pourtant à une destination privilégiée de l'émigration clairacaise ${ }^{38}$. Il n'en continue pas moins de trafiquer avec l'Amérique. Et Marc Fraissinet \& Cie fait de même. Il se livre notamment aux mêmes types d'affaires deux décennies plus tard avec son vaisseau le Saint-Marc-et-Saint-Philippe ${ }^{39}$.

L'armement maritime constitue presque un complément naturel de leur activité, notamment parce qu'il leur permet de viser des marchés que leurs transporteurs habituels du Nord de l'Europe ne fréquentent pas. Les Fraissinet le pratique depuis leurs débuts. Dès les années 1720, les registres de l'amirauté de Sète enregistrent leurs prises de participations sur de nombreux bâtiments. Parfois, ils se font véritablement armateurs lorsqu'ils fournissent aussi la mise de fonds nécessaire au voyage du bâtiment, comme dans le cas de la tartane Sainte-Rose en $1734^{40}$. D'autres fois, ils se contentent de participer à des expéditions organisées par d'autres comme avec le vaisseau Jeune-Jean en $1763^{41}$. Marc Fraissinet donne cependant à son activité d'armateur une impulsion toute particulière au cours des années 1760 qui le fait bientôt désigner comme «négociant et armateur ». Il fait notamment construire à Sète en 1771 un bâtiment d'assez forte portée, le Saint-Marc-et-Saint-Philippe, dont on a vu qu'il le destine à une série d'expéditions à l'Amérique ${ }^{42}$. Sept ans plus tard, ses parts de navires se répartissent sur une dizaine de coques, dont six tartanes et quatre unités plus importantes ${ }^{43}$. Son profil contraste assez nettement avec celui de Jean Balguerie dont les intérêts maritimes sont restés plus limités et ne comportaient quatorze ans plus tôt, au moment de son décès, qu'un quart du pinque 
Jean-Pierre, un bâtiment qu'il venait d'expédier à Saint-Domingue avec retour à Marseille ${ }^{44}$ Balguerie en dehors du commerce des alcools ne contribuent guère à réduire leur exposition aux risques du marché des liquides. Or, celui-ci connait des mouvements conjoncturels violents, liés aux soubresauts du commerce maritime et aux variations de la consommation. Aussi les grandes pulsations de leurs affaires sont-elles dépendantes du contexte international qui influence le climat économique autant qu'il entrave éventuellement la navigation. La plupart de leurs arrêts, à savoir deux des trois faillites Fraissinet, survenus des années 1730 aux années 1770, et le dépôt de bilan de Jean Balguerie intervenu en 1762 sont liées à un état de guerre ${ }^{53}$.

Elles peuvent même apparaître comme des étapes d'un processus de consolidation, lorsqu'elles se concluent par un concordat allégeant le fardeau de leurs dettes. C'est le cas du premier arrêt Fraissinet, consécutif à la faillite du fermier général Pierre Bragouze en $1734^{54}$, et de leur deuxième arrêt, en septembre 1763.

L'année précédente Jean Balguerie avait également succombé dans une conjoncture déprimée liée à la guerre de sept ans, qui frappa un très grand nombre de négociants languedociens. Pierre Lagarde, l'un de ces faillis, a décrit les mécanismes de cette crise : «la dureté du temps et des guerres » s'est traduit, écrit-il, par une grande quantité de marchandises et autres effets détenus à l'étranger restés « invendus en bonne partie » ou qui se vendent « en grosses pertes $»^{55}$. Jean Balguerie obtint un concordat en mars 1763.

Liame, 25 | 2012 
Mais son décès en juillet de l'année suivante empêcha qu'il en honore les termes, et sa maison disparut avec lui ${ }^{56}$.

On a vu que Marc Fraissinet avait dépassé cette phase et poursuivi ses affaires. Il fut contraint à un nouvel arrêt en novembre 1778, lorsque des "pertes considérables ", sans doute liées à l'engagement français dans la guerre d'indépendance américaine, se conjuguèrent au « discrédit » dans lequel le mirent certains de ses créanciers ${ }^{57}$. Il obtint à nouveau un accord avec ses créanciers mais se révéla incapable d'y faire face, si bien qu'il dut négocier un second concordat, qui le mit dans l'obligation de leur abandonner l'ensemble de ses biens-fonds ${ }^{58}$. Contraint de vendre ses magasins, il cessa ses opérations sétoises ${ }^{59}$.

\section{Inscrites dans des trajectoires différentes}

Proches dans leurs formes d'organisation et d'activité, les maisons Fraissinet et Balguerie de Montpellier-Sète prennent place dans des trajectoires familiales dissemblables. Les origines et les postérités de leurs parcours bas-languedociens diffèrent radicalement Elles s'inscrivent pourtant dans les cheminements de deux familles protestantes ayant en commun leur établissement amstellodamois au début du xvIII ${ }^{e}$ siècle. Elles traduisent des comportements voisins en ce sens qu'ils comportent un choix évident de mobilité qui complète, plus qu'il ne s'y oppose, leurs stratégies de sédentarité ${ }^{60}$.

\section{Deux familles protestantes établies à Amsterdam}

À Montpellier, vers 1750, l'ancienneté de la maison Fraissinet contraste avec la nouveauté de l'établissement de Jean Balguerie. Un siècle plus tôt, les deux familles appartenaient à des milieux professionnels et à des espaces géographiques que tout séparait. François Fraissinet vivait à Mauguio, petite ville située à une dizaine de kilomètres à l'est de Montpellier, où il était maître-menuisier, tandis que Jean Balguerie était un marchand de Clairac, une cité de l'Agenais établie à la confluence du Lot et de la Garonne ${ }^{61}$. De Mauguio, Isaac Fraissinet, fils de François, s'était établi à Montpellier, la grande ville voisine qui offrait de meilleures possibilités d'ascension sociale. Là, il ajoutait le commerce du bois à son activité de menuisier et de charpentier ${ }^{62}$. Et il émancipait précocement ses quatre fils afin de leur permettre de développer leurs propres affaires. Marc, son aîné, poursuivit son commerce, tandis que son frère Jacques devint marchandquincailler et que leurs cadets, Isaac et Adrien, firent d'abord profession de marchandsmangonniers, c'est-à-dire de marchands-forains. Dans le même temps, les Balguerie étaient restés dans le Clairacais où leur réussite économique leur permit d'affermir leurs positions sociales par l'achat de terres et de seigneuries, dont le nom, adjoint à leur patronyme, servait à les distinguer ${ }^{63}$.

Après la révocation de l'Édit de Nantes, l'appartenance au protestantisme des Fraissinet et de Balguerie contribuait à rapprocher les trajectoires de certains d'entre eux engagés dans les voies du commerce. Le refuge amstellodamois fut leur destination privilégiée, aussi bien pour les Balguerie, avec Daniel Balguerie ou de son cousin Pierre Balguerie, que pour les Fraissinet, avec Adrien Fraissinet ${ }^{64}$. Daniel Balguerie et Adrien Fraissinet s'y établirent de façon quasi-simultanée vers 1710. 

quatre fils d'Isaac Fraissinet nés entre 1667 et 1675 correspondait la génération des dix arrières-petits-fils de Jean Balguerie, nés de 1662 à 1694 (voir figure $n^{\circ} 2$ ). Or ceux-ci ne s'étaient pas seulement réfugiés à Amsterdam; ils s'étaient aussi assez largement dispersés dans toute l'Europe protestante. En dehors des Provinces-Unis, on les rencontre avant 1750 en Suisse, à Londres, à Hambourg, en Suède et en Prusse ${ }^{65}$. Certains avaient quitté l'Agenais pour des destinations moins lointaines, situées à l'intérieur du royaume, et notamment pour ses ports où leurs aspirations mercantiles trouvaient à se conjuguer avec leur désir d'anonymat religieux. Ils empruntèrent pour cela les mêmes chemins que les autres protestants de Clairac, emportant avec eux leur savoir-faire commercial, là où nombre de leurs compatriotes n'avaient guère que leurs compétences de tonneliers à faire valoir ${ }^{66}$. Ces chemins les conduisirent en priorité vers Bordeaux et, secondairement, vers le Bas-Languedoc, à l'autre extrémité de l'axe commercial qui traverse l'isthme aquitain en passant par leur ville ${ }^{67}$. Un Balguerie est signalé de façon fugace comme négociant agathois en $1718^{68}$. Le plus grand nombre s'établit durablement dans le port de la Lune. Jean Balguerie aîné, son cousin germain Jacques Balguerie et Pierre Balguerie cousin étaient marchands ou négociants à Bordeaux ; ils relevaient de branches familiales distinctes, séparées une ou deux générations plus tôt, qui maintenaient leur cohésion familiale par un jeu complexe d'alliances matrimoniales croisées : une tante de Pierre Balguerie avait par exemple épousé un frère de Jacques tandis que Pierre s'était uni avec une sœur de Jean.

Chez eux comme chez les Fraissinet, l'opportunité d'établir des liens avec un parent installé à Amsterdam fut probablement à l'origine de leur passage des rangs du commerce à ceux du négoce. À Bordeaux, Jacques Balguerie s'appuyait sur l'activité de ses frères Moïse et Daniel. Et les affaires amstellodamoises de Pierre Balguerie allaient de pair, dans le port girondin, avec celle de Pierre Balguerie $\operatorname{cousin}^{69}$. Parmi les Fraissinet, l'établissement d'Adrien à Amsterdam correspondit à celle de son frère Isaac à Sète, d'où il chargeait pour le grand emporium du Nord ${ }^{70}$. Isaac Fraissinet devint ainsi le premier négociant bas-languedocien de sa famille ${ }^{71}$. Son fils Marc rejoignit Adrien aux ProvincesUnies à la fin de la décennie 1720, dans un établissement qui réaffirmait l'importance du lien noué avec le Nord, tandis que ses deux autres fils, Antoine et Isaac restaient auprès de lui, à Sète et à Montpellier ${ }^{72}$. 
Figure $n^{\circ} 2$ : Généalogique de quelques représentants de la famille Balguerie établis dans le commerce au XVIII ${ }^{\mathrm{e}}$ siècle

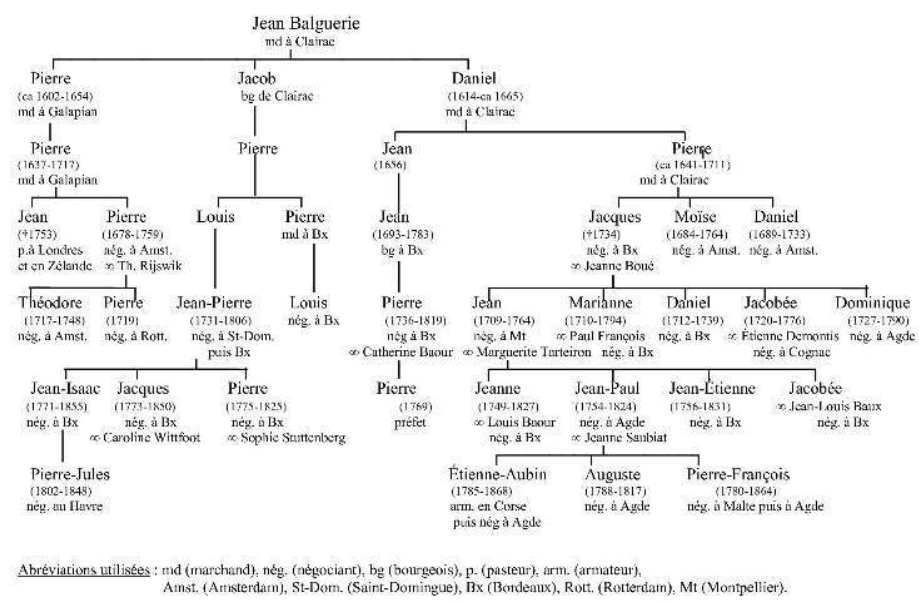

Leur commune présence amstellodamoise des deux familles ne suffit pas à rapprocher les réseaux préexistants aux développements de leurs maisons de commerce baslanguedocienne. Ceux-ci présenteraient même plutôt des morphologies opposées où l'enracinement local, -montpelliérain-, des Fraissinet contraste avec le caractère plus diversifié du réseau Balguerie qui, à partir de Clairac, s'est plus largement répandu tant à Bordeaux que dans l'Europe protestante. Par-delà l'échec de leurs implantations à Montpellier et Sète, un tel écart initial explique les cheminements divergents empruntés par les deux familles car, tandis que le centre de gravité de l'activité Fraissinet bascule du Bas-Languedoc à Marseille, celui des Balguerie conserve sa logique essentiellement bordelaise.

\section{Les Fraissinet de Montpellier à Marseille}

Considérée sur plus d'un siècle, la dynamique des implantations commerciales des Fraissinet se déplace de Montpellier à Marseille (voir figure n 3). Après 1800, la présence familiale dans le chef-lieu de l'Hérault s'interrompt au moment où le port phocéen accueille les enfants de Marc Fraissinet.

Ce passage est facilité par une pratique résolue de mobilité, clairement visible chez les quatre générations de descendants d'Isaac Fraissinet, qui conduit à l'installation de l'un au moins des fils de chaque génération dans un espace commercial distinct de celui de son père. Cette stratégie de développement des affaires familiales passe d'abord par des implantations non-languedociennes. Elle insiste d'abord sur Amsterdam, lieu d'établissement d'un fils et d'un petit-fils d'Isaac Fraissinet. Puis, alors que cette implantation amstellodamoise apparait consolidée par l'association du dernier arrivé avec son propre fils, elle se porte sur Marseille où Jean Fraissinet fait souche et d'où ses 
descendants investissent à leur tour d'autres espaces, essentiellement en Méditerranée occidentale (Livourne, Alger, la Bosnie).

Notons que jusqu'à l'arrêt définitif de la maison Fraissinet de Sète, les maisons Fraissinet d'Amsterdam et de Marseille lui restent très proches, l'étroitesse de leurs relations se manifestant par la corrélation de leurs difficultés en 1763 et 1778. Dans le premier cas, la faille de Marc Fraissinet correspond à celle de la maison marseillaise Veuve Deveer \& Fraissinet. Dans le second, elle est liée à celle de Marc Fraissinet fils à Amsterdam, ce qui fait dire à Fernand Braudel qu'il s'agit d'une faillite hollando-sétoise ${ }^{73}$. Autre signe de cette proximité, après sa liquidation de 1778, Marc Fraissinet trouve dans le port phocéen une position de repli où il retrouve son statut de négociant aux côtés de son frère. Mais cette solution reste pour lui transitoire car il se réinstalle en Bas-Languedoc au début de la Révolution. De retour à Montpellier, il se livre à nouveau au commerce jusqu'à son décès intervenu au début du Consulat ${ }^{74}$.

À ce moment, deux de ses fils restent encore apparemment attachés à la ville : Joseph y habite toujours, tandis que Jean-Louis continue d'un résider " ordinairement». Mais le premier s'installe ensuite à Paris tandis que le second a déjà ses affaires à Marseille. Leur frère Pierre s'est pour sa part établi aux Indes au début de la décennie 1790. À Chandernagor, il codirige avec le montpelliérain Eustache Vialars une filiale de la maison marseillaise Rabaud. Puis il crée à Calcutta une nouvelle maison, connue sous la raison Grillard Fraissinet \& Cie, qui sert notamment de correspondant en 1793 au capitaine $\mathrm{Grove}^{75}$. Revenu en France, il s'installe enfin à Marseille. Dès lors, les Fraissinet ne figurent plus dans les rangs du négoce montpelliérain de la première moitié du XIX siècle. Il faut attendre l'arrivée d'Adolphe Fraissinet comme trésorier payeur général du département pour voir réapparaitre leur patronyme dans le monde des affaires locales, une arrivée par ailleurs facilitée par le crédit que lui offrent ses parents montpelliérains qui versent le cautionnement de son emploi, dans un geste qui témoigne de la vitalité du réseau qui lie alors nombre de familles négociantes protestantes à cheval sur Montpellier et Marseille. 


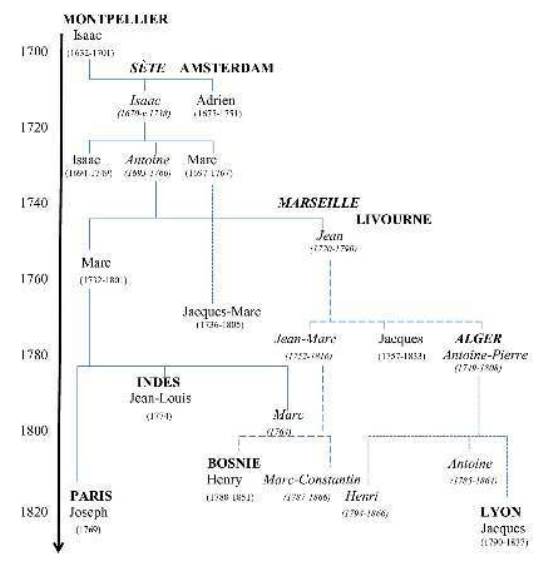

Note : tous les individus cités sont négociants à l'exception du premier, Isaac, marchand de bois.

\section{Les Balguerie dans une logique girondine}

40 Les implantations commerciales des Balguerie, même limitées aux enfants de Pierre Balguerie du Metge, font de Bordeaux et, très secondairement, d'Agde, les pivots de leurs entreprises familiales. Car un membre de chaque génération des descendants de Pierre est représenté dans le port de la Lune (voir figure $n^{\circ} 4$ ). Et, même lorsqu'ils font souche à Agde, la logique de leurs installations ressort essentiellement des impératifs du commerce girondin.

41 Cette logique d'établissements s'inscrit dans un mouvement assez voisin dans son principe de celui qui anime les Fraissinet puisque, après avoir assuré leur présence à Bordeaux, des enfants de chaque génération partent au loin, d'abord en Hollande, puis à Marseille, mais pour peu de temps car Jean Balguerie préfère finalement Montpellier au port phocéen. L'installation à Agde, déjà essayée au début de la Régence, peut-être par un oncle de Jean Balguerie, est reprise au cours des années 1760 ou 1770 par Dominique, le frère de Jean. Là, les Balguerie trouvent un nouveau terme à leurs cheminements méditerranéens et ils font souche par l'intermédiaire de deux des fils de Jean Balguerie.

L'établissement de Jean Balguerie en Bas-Languedoc intervient au terme d'un processus qui le voit rechercher dans le Midi méditerranéen les conditions d'installation les plus favorables à son activité. Il a probablement débuté sa carrière commerciale auprès de l'un de ses parents, probablement chez son père à Bordeaux ou peut-être chez l'un de ses oncles établis aux Provinces-Unies. Après le décès de son père en 1734, il a peut-être 
repris la maison de commerce paternelle en compagnie de son frère cadet, Daniel, décédé dès 1739 , mais rien n'est assuré en l'état actuel de la recherche.

On saisit en revanche avec certitude ses efforts pour s'établir dans le Midi au cours de la décennie suivante. Il participe tout d'abord jusqu'en 1745 aux affaires d'une maison située à Montréal, petite bourgade située le long du canal des deux mers, au sud-ouest de Carcassonne $^{76}$. Là, il a probablement assuré le même type de commerce de transit que celui dont ses aïeuls clairacais se chargeaient déjà, et particulièrement des draps de laines produits autour de Carcassonne ou descendus du Castrais tout proche.

Il s'établit ensuite à Marseille où il fréquente des négociants dont les origines ont à voir avec son implantation précédente, et notamment les Audibert venus du Castrais. Vers la même époque, il paraît nouer des liens avec le négociant sétois Louis Rogé avec qui il partage des origines géographiques communes. Rogé est en effet né à Clairac, lieu de naissance du père de Jean Balguerie, et il a grandi à Bordeaux ${ }^{77}$. Arrivé à Sète vers 1725 en tant que tonnelier, on a vu qu'il s'y était associé à Verborg, l'un des principaux exportateurs sétois d'eau-de-vie du début de la décennie $1730^{78}$. Le départ de ce dernier pour Hambourg vers 1743 l'a laissé dans la position de prendre un nouvel associé afin de donner une nouvelle impulsion à ses affaires ${ }^{79}$. Il crée une nouvelle société avec Balguerie vers 1746, date à partir de laquelle il fait transcrire dans les registres de l'amirauté de Sète ses manifestes de chargements de navires au nom de Rogé \& Balguerie. Dans l'association, Balguerie présente trois séries d'atouts : il dispose tout à la fois de capitaux, d'une expérience commerciale et de relations étendues ${ }^{80}$. La mise en relation des deux hommes est peut-être passée par le frère de Louis Rogé, actif dans le port girondin, à moins qu'elle ne résulte d'autres biais, à l'image des relations nouées entre les Laurens de Montpellier-Sète et les Roux de Marseille par l'intermédiaire d'Amsterdam. En effet, le négoce sétois travaillait avec certains des Balguerie de Hollande, comme les Laurens qui comptaient parmi leurs clients Jean Balguerie Junior, de Rotterdam, fils du négociant amstellodamois Pierre Balguerie, qui n'était lui-même pas un parent bien éloigné du Jean Balguerie qui nous intéresse.

Associé à Louis Rogé, Jean Balguerie réside quelques années à Marseille ${ }^{81}$. Leur société, à cheval sur le Bas-Languedoc et la Provence, s'inscrit dans une configuration qui, sans être totalement originale, n'est pas très courante, et que l'on ne rencontre que dans quelques cas de sociétés dirigées par des étrangers ${ }^{82}$. Cette organisation cesse cependant avec l'établissement de Balguerie à Montpellier, attesté à partir de $1749^{83}$.

Son frère Dominique Balguerie l'a d'abord suivi à Montpellier avant de repartir à Bordeaux au cours des années $1750^{84}$. Il revient en Bas-Languedoc pour s'établir à Agde, probablement lors de la décennie suivante. Il s'agglomère alors au commerce agathois, dans une logique commerciale déjà plusieurs fois empruntée par la famille, notamment à dans le port, un demi-siècle plus tôt, et à Montréal, au début de la décennie 1740, où il s'agit à chaque fois de participer aux trafics de transit passant par le canal du Midi dont Agde est le débouché méditerranéen et Bordeaux l'ouverture atlantique.

47 À Agde, la rupture de charge entre les barques de canal et les bâtiments de mer génère plusieurs types d'affaires. Les maisons de commerce s'y occupent des blés et des produits coloniaux empruntant le canal: elles réceptionnent les céréales venues du HautLanguedoc et la partie du sucre et du café que Bordeaux destine à Marseille par la voie intérieure $^{85}$. En sens contraire, elles expédient vers le port girondin les produits provençaux et languedociens, agricoles -à l'image des vins-, ou industriels, -avec les draps, les soieries ou le savon. En période de disette, elles font aussi faire remonter dans 
l'isthme aquitain les blés achetés en Méditerranée ${ }^{86}$. Leur activité n'est pas seulement commerciale et elle comporte une part importante d'armements car elles dominent la liaison avec Marseille qu'assurent leurs tartanes ${ }^{87}$. L'importance de leurs affaires navales se marque aussi par la formation de capitaine qu'elles donnent couramment aux enfants de leurs dirigeants.

Dominique Balguerie ne se comporte pas différemment des autres négociants d'Agde. Associé à l'agathois Gabriel Carriès sous la raison Carriès \& Balguerie, ses affaires ne le distingue pas des autres maisons locales, ni dans les produits qu'il traite, ni dans la préparation qu'il donne à ses neveux destinés à prendre sa suite. C'est ainsi que JeanÉtienne Balguerie, plus connu sous le nom de Balguerie junior, opte pour le métier de capitaine. On a déjà signalé son brillant destin dans le port de la Lune, où il exerce ses talents de marin avant d'y devenir négociant et armateur ${ }^{88}$. Son activité y est trop bien connue pour devoir être rappelée. La même formation de capitaine est donnée par la suite aux fils de son frère aîné, Jean-Paul, dit Balguerie neveu, continuateur de la maison de commerce agathoise après le décès de son oncle.

Balguerie neveu y est associé à Jean-Séver Pioch, lui-même fils d'un capitaine du port, sous la raison Balguerie \& Pioch. Les relations qu'il entretient avec son frère installé à Bordeaux ne sont pas seulement d'ordre marchand et ne se limitent pas à sa fourniture des toiles d'Agde ${ }^{89}$. Elles se traduisent également par l'identité de leurs comportements car l'un et l'autre se livrent à l'armement, au commerce et à la course ${ }^{90}$. Entre 1809 et 1813, Balguerie \& Pioch arment ainsi le plus fort corsaire marseillais de la période, le trois-mâts La Babiole qui part à trois reprises du port phocéen mais aussi de Sète et de Toulon $^{91}$. Et en 1812, l'entreprise agathoise fait franciser à Sète un bâtiment venu de prises, le brick Le Commerce ${ }^{92}$. Son négoce s'étend largement en Méditerranée avec deux des fils de Balguerie neveu établis respectivement armateur en Corse et négociant à Malte.

Sous la Restauration, leur retour semble signifier le recul des affaires Balguerie d'Agde et leur repli sur le port du fleuve Hérault, où ils deviennent pourtant des notables, et où ils occupent les fonctions de maire de 1830 à 1834 et de vice-consul du Danemark. Leur situation souffre cependant de la comparaison avec celle de leur oncle Balguerie junior qui est alors devenu l'un des plus influents négociants bordelais. Avec lui, Bordeaux est redevenu l'implantation commerciale la plus importante des Balguerie. 
Figure 4 : Les dynamiques spatiales des implantations commerciales des Balguerie issus de Pierre Balguerie du Metge

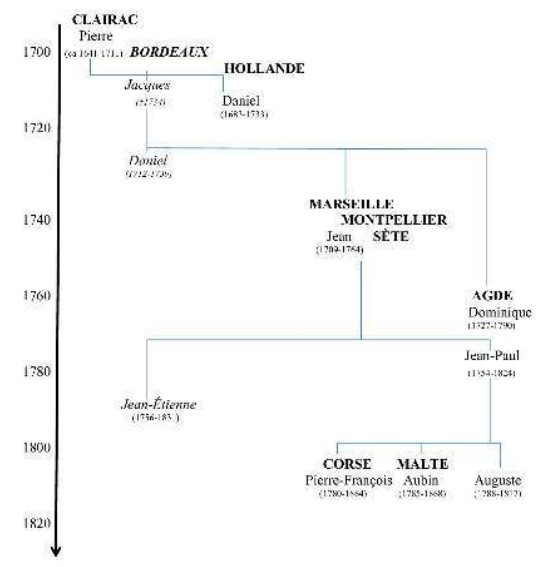

$51 \mathrm{Au}$ terme de leurs cheminements bas-languedociens, qui les ont fait se côtoyer à Montpellier et à Sète, les Fraissinet et les Balguerie trouvent ou retrouvent à Marseille et à Bordeaux l'horizon principal de leurs affaires. Ils y sont solidement implantés au début de la Révolution avec deux de leurs représentants natifs de Montpellier, une réussite qui illustre leur capacité à rebondir et qui traduit l'existence des réseaux qu'ils ont su y faire jouer depuis Montpellier ou Sète ${ }^{93}$.

\section{Fondatrices de réseaux centrés sur le Bas-Languedoc}

52 L'appréhension de leurs réseaux centrés sur le Bas-Languedoc se fonde ici sur l'examen de leur composante familiale. Leurs liens de parenté ne suffisent évidemment pas à épuiser la diversité de réseaux mais ils en constituent une composante essentielle et ils offrent en outre l'avantage de mobiliser des sources disponibles puisqu'il s'appuie sur l'examen de leurs principaux actes d'état-civil. Ceux-ci présentent leurs différentes parentés, qu'elles soient formelles et nouées à l'occasion de mariages, ou spirituelles et formées lors du baptême des enfants. Ils renseignent aussi sur la partie la plus importante de leur environnement local non familial, engagée comme témoins ou simples participants à ces actes majeurs de leur vie sociale ${ }^{94}$. Le comptage des individus intervenant lors de leurs mariages et de leurs baptêmes permet de dessiner à la fois la morphologie et la dynamique de leurs réseaux, depuis leur mise en place jusqu'à leur déstructuration. Il permet aussi d'envisager l'existence d'un modèle de réseau partagé par les Fraissinet et les Balguerie, à travers la recherche d'éventuelles logiques communes ayant présidé d'abord à leur construction et à leur réassurance. 


\section{Des dynamiques de mise en place communes}

53 La formation des réseaux sur lesquels s'appuient leurs maisons de commerce baslanguedociennes du milieu $d u x_{\text {XVIII }}{ }^{e}$ siècle, présente des chronologies étroitement parallèles : après le déploiement des deux familles aux Provinces Unies, dont on a déjà l'étonnante simultanéité, les Fraissinet et les Balguerie affirment leurs liens avec Marseille qui constituent, vers 1746-1748, le second facteur déterminant de leurs réussites.

Jean Balguerie s'unit dans le port phocéen en juillet $1748^{95}$. Il y négociant, résidant sur le Cour, et associé de la maison Rogé \& Balguerie également présente à Sète. Son mariage s'inscrit au cœur du grand négoce protestant marseillais car il réunit autour de lui plusieurs de ses principaux membres. L'évènement se déroule en effet chez Isaac Tarteiron, l'un des plus importants négociants et armateurs de la place, en présence notamment des négociants Antoine Hugues ${ }^{96}$, gendre d'Isaac Tarteiron, et de François Isnard $^{97}$. Isaac Tarteiron est un parent de Marguerite Tarteiron, l'épouse de Jean Balguerie : il est le grand-oncle de son père. Il partage avec elle son lieu de naissance dans la petite ville cévenole de Ganges ${ }^{98}$. L'importance de la dot qu'il lui verse traduit l'intérêt qu'il porte à son union. Son mariage forme en effet comme un trait d'union entre les négoces bordelais et marseillais D'ailleurs, c'est François Isnard, négociant en liens avec le port de la Lune, qui représente la mère de Jean Balguerie lors de sa célébration ${ }^{99}$. Ajoutons que le négoce montpelliérain y a également une place du fait de la présence de Jean Tarteiron, oncle de l'épouse, une place limitée mais décisive car le nouveau ménage s'installe l'année suivante à Montpellier où Balguerie, Rogé et ce Jean Tarteiron créent ensemble leur entreprise pour le commerce à Saint-Domingue ${ }^{100}$.

Les Fraissinet sont également présents à Marseille en 1748 avec Jean Fraissinet, le frère de Marc. Son implantation y concrétise des relations anciennes, entretenues depuis le début du siècle, notamment par Adrien avant son émigration à Amsterdam ${ }^{101}$. Jean est négociant associé aux frères Pierre et Henry Deveer, deux amstellodamois avec qui il partage la direction de Deveer frères \& Fraissinet. Sa firme renforce des liens préexistants entre la maison Antoine Fraissinet de Montpellier et la maison A. Deveer d'Amsterdam ${ }^{102}$. Forte de ces relations, elle connaît une réussite rapide et elle figure dès le début de la décennie 1750 aux premiers rangs du commerce marseillais. Ses affaires s'étendent en Méditerranée occidentale au port franc de Livourne où Jean Fraissinet s'installe quelques années vers 1756-1758. Localement, elle est en liens avec d'autres négociants protestants, comme les frères David et Roger Roux, présents en mai 1749 lors du mariage de Jean Fraissinet avec sa cousine-germaine Marguerite Fraissinet, fille d'Isaac ${ }^{103}$. Elle noue également des relations avec le Jean Baux, négociant marseillais d'origine Castraise, qui épouse en juillet 1750 Constance Fraissinet, la sœur de Marc et de Jean. En la circonstance, Marguerite Fraissinet se voit doter de $20.000 \mathrm{ft}$ de la part de ses parents et de $10.000 \mathrm{ft}$ de Pierre Deveer, chez qui est signé le contrat mariage ${ }^{104}$. Mais l'intérêt de la maison Fraissinet de Montpellier-Sète n'est pas absent de ces unions car on a vu que par la suite les frères Jean et David Baux s'associent à Marc Fraissinet dans plusieurs de entreprises coloniales. Ils constituent d'ailleurs en 1778 ses principaux correspondants marseillais ${ }^{105}$.

Vers 1750, les réseaux des parents proches de Marc Fraissinet et de Jean Balguerie couvraient facilement les trois espaces amstellodamois, montpelliérain et marseillais 
(voir tableau n 1). Celui de Balguerie s'étendait aussi sur Bordeaux et Hambourg tandis que celui de Fraissinet attend son second mariage, en 1762, pour atteindre le port girondin ${ }^{106}$.

La densité des liens locaux de Marc Fraissinet est la contrepartie de leur moindre développement géographique ${ }^{107}$. Elle s'explique par l'ancienneté de l'implantation montpelliéraine de sa famille et elle rend compte de son appartenance aux milieux d'affaires réformés de la ville. Son mariage en 1753 avec Suzanne Corbière Larigaudier, fait par exemple de lui le beau-frère par alliance d'Étienne Périer et d'Antoine Seimandy, soit deux des plus importants négociants protestants de la place, tous deux liés à Marseille ${ }^{108}$. Pour lui comme pour son père, ces unions contractées localement se doublent d'enjeux financiers importants car les apports dotaux des épouses sont systématiquement absorbés par l'entreprise, et confondus avec leurs capitaux pour faire fonds dans la société familiale ${ }^{109}$. Antoine, père de Marc, reçoit ainsi $10.000 \mathrm{ft}$ en 1718 . Et il dote son fils Marc de $20.000 \mathrm{ft}$ en 1753, une somme qui double celle reçue par sa bru, et qui élève l'apport social de Marc à un tiers d'intérêt dans la société dans laquelle il rentre aux côtés de son père ${ }^{110}$. Les relations nouées à l'occasion de mariages contribuent aussi à la solidité de l'entreprise familiale, non seulement par le biais de participations, par exemple dans le domaine de l'armement maritime, mais aussi par l'appui qu'elles offrent en cas de difficulté. Ainsi, en 1734, mis à mal par la faillite Bragouze, la maison Fraissinet père \& fils obtient-t-elle un crédit de $7.500 \mathrm{ft}$ de Veuve Sarran \& Bazille avec qui elle est déjà en compte pour la vente de certaines marchandises ${ }^{111}$. Quarante ans plus tard, les principaux créanciers de la faillite Fraissinet de 1778 relèvent tous plus ou moins étroitement du cercle familial.

Localement, les Balguerie ne peuvent pas s'appuyer sur un réseau aussi dense. Du temps de Jean Balguerie, leurs relations familiales à Montpellier se réduisent à Jean Tarteiron, l'oncle de son épouse. Elles sont en revanche très importantes à Bordeaux où Jean Balguerie est dans les années 1750 le neveu de Dominique Rion, le frère de Dominique Balguerie, et le beau-frère de Paul François et d'Étienne Demontis, sans compter nombre de parents Balguerie moins proches. Tous y sont négociants ${ }^{112}$. Les Balguerie n'ignorent pas pour autant le berceau géographique de la famille puisque Jeanne-Éléonore Balguerie, autre sœur de Jean, épouse Pierre Espaignac aîné, un marchand de Clairac, et parce que Jean Balguerie y devient ensuite en 1760 le parrain de sa nièce Jeanne Espaignac. C'est d'ailleurs à Clairac que son frère Dominique choisit de finir ses jours trente ans plus tard ${ }^{113}$. Dernier espace original de son réseau familial, ils possèdent des parents à Hambourg où son oncle Pierre Boué (1677-1745) est devenu l'un des principaux trafiquants de produits coloniaux ${ }^{114}$.

Tableau. $\mathbf{n} 1$ : Réseaux de parentés proches de Marc Fraissinet et de Jean Balguerie entre 1740 et 1765 (pères, beaux-pères, oncles, oncles par alliance, frères, beaux-frères et cousins germains)

\begin{tabular}{|c|c|c|c|c|c|}
\hline & Montpellier & Bordeaux & Marseille & Amsterdam & Hambourg \\
\hline $\begin{array}{l}\text { Marc } \\
\text { Fraissinet }\end{array}$ & $\begin{array}{lr}\text { Isaac F., } & \text { Antoine F., } \\
\text { Daniel } & \text { Corbière- } \\
\text { Larigaudié, } & \text { Étienne } \\
\text { Périer, } & \text { Antoine } \\
\text { Seymandy, } & \text { Pierre } \\
\text { Arnaud. } & \end{array}$ & Joseph Arnaud & $\begin{array}{l}\text { Jean } F . \\
\text { Jean } \\
\text { Baux. }\end{array}$ & $\begin{array}{l}\text { Marc F., } \\
\text { Jean-Marc } \\
\text { F. }\end{array}$ & \\
\hline
\end{tabular}




\begin{tabular}{|l|l|l|l|l|l|}
\hline $\begin{array}{l}\text { Jean } \\
\text { Balguerie }\end{array}$ & Jean Tarteiron. & $\begin{array}{l}\text { Dominique Rion, } \\
\text { Dominique B., Paul } \\
\frac{\text { François, Étienne }}{\text { Demontis. }}\end{array}$ & Moïse B. & $\begin{array}{l}\text { Pierre } \\
\text { Boué }\end{array}$ \\
\hline $\begin{array}{l}\text { Note: tous les individus cités sont des négociants. Ceux de la génération des frères, beaux-frères et cousins sont soulignés. Ceux rentrés } \\
\text { dans le réseau familial par des mariages postérieurs à 1750 sont indiqués en gras. }\end{array}$
\end{tabular}

\section{Des fonctionnements voisins}

59 L'examen des liens de parentés découlant de la naissance et du mariage des enfants de Marc Fraissinet et de Jean Balguerie dessine, à travers le choix de leurs parents spirituels, parrains et marraines, et de leurs époux et épouses, les espaces de leurs réseaux sociaux ${ }^{115}$ . Comparé aux espaces de leurs réseaux préexistant au développement de leurs maisons de commerce, il montre comment ces réseaux se réassurent dans le temps en établissant de nouveaux liens (voir tableau $n^{\circ} 2$ ).

60 Les baptêmes des enfants Marc Fraissinet et de Jean Balguerie sont célébrés entre 1749 et 1778, soit au cours de la période d'activité de leurs firmes. Chacune des deux fratries fait ressortir un espace privilégié ainsi que trois ou quatre espaces secondaires, mais les deux ont en commun de couvrir les trois grands espaces commerciaux du Midi : Montpellier/ Sète, Bordeaux et Marseille.

61 Les baptêmes des enfants de Marc Fraissinet sont essentiellement célébrés avec des parrains de Montpellier ou de Sète (aux deux-tiers). Tous leurs sont apparentés, même Jean-Louis Engelke, époux de Marguerite Boischon. Leurs trois autres origines de leurs parrains sont Amsterdam, Bordeaux et surtout Marseille où Marc Fraissinet a choisi trois d'entre eux : son frère Jean, sa sœur Constance et le négociant Jean Roux. Ce dernier, qui ne présente pas de liens de parenté a priori, s'inscrit dans le milieu du grand négoce protestant marseillais aux côtés de Jean Baux ou de Jacques Seymandy qui, eux, sont bien ses parents plus ou moins éloignés.

Pour Jean Balguerie, le choix des parrains et marraines privilégie Bordeaux dans six cas sur treize (38\%). Il prend soin de donner à presque tous ses enfants un parrain ou une marraine dans le port de la Lune, sans que celui-ci ait la même centralité que Montpellier pour le réseau de Marc Fraissinet. Sur les bords de la Garonne, il fait appel à sa mère, à son frère, à deux de ses sœurs et à d'un de ses beaux-frères, le négociant Étienne Demontis. Ceux qu'il choisit à Montpellier ou à Sète forment $29 \%$ des parrains de ses enfants. Ils n'ont pas de relations de parenté avec lui et ne présentent pas le standing social des parents spirituels des enfants de Marc Fraissinet: Jean-Jacques Fargeon, marchand-parfumeur de Montpellier, et Anne Carabasse, son épouse, interviennent longtemps dans leurs actes de baptêmes en tant que représentant des parrains et marraines avant d'être choisi pour tenir ce rôle. À Sète, Jean Balguerie fait appel à Laurent Martin, le capitaine marin qui commande son pinque Jean-Pierre, et à son épouse Rose Bonnet $^{116}$. À Marseille, son choix est plus élitiste puisque Jean Tarteiron, est à la fois un parent de son épouse, fils d'un cousin d'Isaac Tarteiron de Marseille, et un négociant du port provençal ${ }^{117}$. À Ganges, il fait classiquement appel aux grands-parents maternels de ses enfants et à Clairac à l'une de ses sœurs. 
63 essentiellement parce qu'une bonne partie d'entre eux ne parviennent pas à l'âge adulte. Ceux qui le font accentuent par le choix de leurs conjoints les tendances perceptibles au moment de leurs naissances. Les deux enfants de Marc Fraissinet dont on connaît les unions se marient pour le garçon à Montpellier, dans une riche famille protestante locale, et pour la fille à Marseille, à un grand négociant du port.

Quant aux quatre enfants mariés de Jean Balguerie, tous convolent à Bordeaux à des négociants ou à des filles de négociants. Ses deux filles Jeanne et Marie-Jacobée épousent les négociants girondins Alexis Baour en 1765 et Jean-Louis Baux en $1778^{118}$. Ses fils Balguerie neveu et Balguerie junior épousent les bordelaises Jeanne Saubiat et Anne Rion, une série d'union qui traduit une évidente volonté d'appartenance aux milieux du négoce protestant bordelais à une époque où Jean Balguerie est décédé et où sa maison de commerce bas-languedocienne n'existe plus ${ }^{119}$. Ces mariages constituent dans la majorité des cas des ré-enchaînements d'alliances qui contribuent à retisser la toile de leurs réseaux familiaux: Alexis Baour est tout à la fois le beau-frère du négociant bordelais Pierre Balguerie et le fils de Pierre Baour et de Toinette Balguerie, une petite-nièce de la tante de Jean Balguerie, née Judith Balguerie de Ramons ${ }^{120}$. Anne Rion est la fille de du négociant Dominique Rion et de Jeanne François, elle-même fille du négociant bordelais Paul François et de Marianne Balguerie, et donc petite-nièce de Jean Balguerie. Lorsque Balguerie Junior se remarie en 1793 dans le Gard, avec Sophie Dupuy-Montbrun d'Aubignac, il s'agit encore d'une alliance nouée dans le cercle des relations familiales, qui rappelle ici son ancienne alliance marseillaise car son épouse est la belle-sœur de l'ancien négociant phocéen Jean Tarteiron déjà évoqué, établi à Bordeaux au cours de la décennie $1760^{121}$. Cette union touche au rôle de passerelle joué par le réseau Balguerie entre Marseille et Bordeaux : dès 1765, Isaac Tarteiron, frère de Jean, s'est marié dans le port de la Lune en présence notamment du négociant bordelais Trapaux aîné, parent des Balguerie. On le retrouve ensuite, vingt ans plus tard, lors du mariage de Paul Duret avec Marguerite Balguerie, la fille de Pierre et de Catherine Baour, et la nièce de Jeanne Balguerie, fille de Jean et de Marguerite Tarteiron ${ }^{122}$.

Le même caractère endogamique concerne aussi au moins l'un des deux mariages des enfants de Marc Fraissinet, lorsque, en 1795, Suzanne Fraissinet épouse son cousingermain Marc-Élisée Baux, le fils de David Baux et de Constance Fraissinet ${ }^{123}$. On ajoutera que le négociant marseillais, Jean Fraissinet, frère de Marc, avait déjà épousé sa cousinegermaine fille d'Isaac Fraissinet de Montpellier.

De telles unions, constamment renouées au sein du même réseau social, eurent des conséquences très concrètes sur le destin des deux familles puisqu'ils renforçaient la présence des Fraissinet à Marseille et parce qu'ils ouvrirent l'opportunité à Balguerie junior de travailler à Bordeaux pour son beau-frère Jean-Louis Baux, un beau-frère dont $\mathrm{il}$ devint l'associé en 1788 avant d'acquérir son indépendance commerciale.

Tableau $\mathrm{n}^{\circ} 2$ : Parentés formelles et spirituelles contractées lors des baptêmes et des mariages des enfants d'Antoine Fraissinet, de Marc Fraissinet et de Jean Balguerie

\begin{tabular}{|l|l|l|l|l|}
\hline \multirow{2}{*}{} & \multicolumn{4}{|l|}{ Patronymes et localisations des parrains / conjoints } \\
\cline { 2 - 5 } & MONTPELLIER/SÈTE & BORDEAUX & MARSEILLE & AUTRES \\
\hline
\end{tabular}




\begin{tabular}{|c|c|c|c|c|c|}
\hline \multirow[t]{2}{*}{$\begin{array}{l}\text { Enfants } \\
\text { de Marc } \\
\text { Fraissinet }\end{array}$} & $\begin{array}{l}8 \text { baptêmes de } \\
1755 \text { à 1778 : } \\
\text { Bernardine, } \\
\text { Pierre-Marc, } \\
\text { Suzanne, } \\
\text { Joseph, Jacques- } \\
\text { Marc, Jean- } \\
\text { Louis, Jean, } \\
\text { Suzanne- } \\
\text { Rosalie }\end{array}$ & $\begin{array}{l}11: \text { Antoine } \\
\text { Fraissinet, } \\
\text { Bernardine } \\
\text { du Caussinel, } \\
\text { Arnaud, } \\
\text { Aoischore } \\
\text { Bouisson, Jeanne } \\
\text { Arnaud, Jean-Louis } \\
\text { Engelke (de Stettin), } \\
\text { Charlotte Martel, } \\
\text { Élisabeth Arnaud, } \\
\text { Pierre Fraissinet, } \\
\text { Suzanne Fraissinet }\end{array}$ & $\begin{array}{l}\text { Joseph } \\
\text { Arnaud }\end{array}$ & $\begin{array}{l}3: \quad \text { Jean } \\
\text { Fraissinet, } \\
\text { Constance } \\
\text { Fraissinet, } \\
\text { Jean Roux }\end{array}$ & $\begin{array}{l}\text { À AMSTERDAM : } \\
\text { Jacques-Marc } \\
\text { Fraissinet }\end{array}$ \\
\hline & $\begin{array}{l}2 \text { mariages en } \\
1795-1796\end{array}$ & Élisabeth Grand & & $\begin{array}{l}\text { Marc-Élisée } \\
\text { Baux }\end{array}$ & \\
\hline \multirow[t]{2}{*}{$\begin{array}{l}\text { Enfants } \\
\text { de Jean } \\
\text { Balguerie }\end{array}$} & $\begin{array}{l}7 \text { baptêmes de } \\
1749 \text { à } 1762: \\
\text { Jeanne, Isabeau, } \\
\text { Jean-Paul, Jean } \\
\text { Étienne, Marie } \\
\text { Jacobée, } \\
\text { Laurent }\end{array}$ & $\begin{array}{l}4: \quad \text { Jean-Jacques } \\
\text { Fargeon, Anne } \\
\text { Carabasse, Laurent } \\
\text { Martin, Rose Bonnet }\end{array}$ & $\begin{array}{l}\text { 6: Jeanne } \\
\text { Boué, } \\
\text { Dominique } \\
\text { Balguerie, } \\
\text { Marie-Jacobée } \\
\text { Balguerie } \\
\text { Suzanne } \\
\text { Balguerie, } \\
\text { Étienne } \\
\text { Demontis }\end{array}$ & $\begin{array}{l}\text { Jean } \\
\text { Tarteiron }\end{array}$ & \begin{tabular}{|l} 
À GANGES : \\
Laurent \\
Tarteiron, \\
Isabeau \\
Léautard \\
À CLAIRAC : \\
Éléonore \\
Balguerie
\end{tabular} \\
\hline & $\begin{array}{l}4 \text { mariages de } \\
1765 \text { à } 1793\end{array}$ & & $\begin{array}{l}\text { Alexis Baour, } \\
\text { Jeanne } \\
\text { Saubiat, Jean- } \\
\text { Louis Baux, } \\
\text { Anne Rion }\end{array}$ & & $\begin{array}{l}\text { À LASSALE : } \\
\text { Sophie } \\
\text { Dupuy- } \\
\text { Montbrun } \\
\text { d'Aubignac }\end{array}$ \\
\hline
\end{tabular}

L'analyse conduite à partir des enfants de Balguerie neveu, le fils de Jean Balguerie établi à Agde, donne évidemment des résultats très différents qui traduisent la destructuration du réseau des Balguerie restés en Bas-Languedoc au moment de la Révolution. Car, même s'il a épousé une bordelaise et s'il entretient des liens commerciaux avec son frère Balguerie junior à Bordeaux, le mariage de tous ses enfants se déroule à Agde ${ }^{124}$. En 1778, sa fille Marie-Jacobée a pourtant bénéficié d'un double parrainage girondin composé de son oncle Balguerie junior, alors capitaine au long-cours, et de sa tante Marie-Jacobée Balguerie. Mais elle constitue une exception, car moins du tiers des parrains de ses enfants sont bordelais (voir tableau n $\left.{ }^{\circ} 3\right)^{125}$. La majorité d'entre eux est issue d'Agde et, en dehors de leur oncle Dominique Balguerie, ne sont pas apparentés à la famille. Dominique Balguerie et son associé Gabriel Carriès dont les deux seuls à être négociants. Les autres sont le maître-tonnelier François Allemand et son épouse Rose Valette, le garçoncordonnier Jean Lauvet et Louise André, servante chez les parents de l'enfant.

Les quatre mariages agathois des enfants de Balguerie neveu s'inscrivent tous dans le milieu du négoce du port même si Magdelaine Méry, l'épouse de Pierre-François 
Balguerie, est née à Marseille ${ }^{126}$. Au terme de cette phase, les Balguerie se sont littéralement fondus dans le milieu des capitaines marins et des négociants agathois. D'ailleurs, ils ne font plus intervenir d'étrangers à la localité dans leurs actes d'état civil. Leur enracinement local s'est effectué au détriment de la composante bordelaise de leurs réseaux.

Tableau n 3 : Parrains et époux des enfants de Jean-Paul Balguerie

\begin{tabular}{|c|c|c|c|}
\hline & Agde & Montpellier & Bordeaux \\
\hline $\begin{array}{l}7 \text { enfants entre } 1775 \text { et } 1788 \text { : } \\
\text { Jean-Gabriel, Jean- } \\
\text { Dominique-Gabriel, Marie- } \\
\text { Jacobée, Pierre-François, } \\
\text { Agathe, Étienne-Aubin, Jean- } \\
\text { Charles-Auguste }\end{array}$ & $\begin{array}{l}\text { 9: Gabriel Carriès, } \\
\text { Dominique Balguerie, } \\
\text { François Allemand, Rose } \\
\text { Valette, Jean Lauvet, Louise } \\
\text { André, Étienne Aubin, Claire } \\
\text { Barthes, Agathe Balguerie }\end{array}$ & $\begin{array}{l}\text { Marguerite } \\
\text { Tarteiron }\end{array}$ & $\begin{array}{lr}4: & \text { Jeanne } \\
\text { Balguerie, Étienne } & \\
\text { Balguerie junior (2 } & \\
\text { fois), } \quad \text { Marie- } \\
\text { Jacobée Balguerie }\end{array}$ \\
\hline $\begin{array}{l}4 \text { mariages entre } 1799 \text { et } \\
1813\end{array}$ & $\begin{array}{l}4: \text { André Prunet, Magdelaine } \\
\text { Méry, Victor Embry, Civique } \\
\text { Bousquet }\end{array}$ & & \\
\hline
\end{tabular}

Figures de proue du commerce méridional pendant plus d'un siècle, les Fraissinet et les Balguerie renouvellent notre vision des réseaux négociants méridionaux. En déplaçant le regard pour examiner leurs réseaux centrés sur le Bas-Languedoc, ils permettent la mise en évidence de dynamiques de construction parallèles et de morphologies relativement proches, essentiellement différenciées par leurs tropismes phocéen et bordelais. Ils révèlent l'existence d'une sorte de modèle de réseau négociant bas-languedocien, dont l'identité se construit à partir d'activités dominées par le commerce des liquides vers le Nord. Ce réseau existe dans une quadruple dimension spatiale qui associe l'échelle locale de Montpellier et de Sète aux échelles régionales de Marseille et de Bordeaux et à l'échelle internationale du nord de l'Europe dominée par le port d'Amsterdam. Son amplitude confère aux familles qui y participent de remarquables capacités de résistance qui leurs permettent de maintenir leur prospérité et, dans les cas qui nous intéresse ici, de se redéployer hors du Bas-Languedoc. À travers lui, les maisons de commerce baslanguedociennes des Balguerie et des Fraissinet montrent la solidarité qui unissait les différents midis de la France dans des réseaux qu'on peut dès lors qualifier de méridionaux ${ }^{127}$.

\section{NOTES}

1. Au milieu du XIX ${ }^{\mathrm{e}}$ siècle, l'importance des deux familles leur vaut de prendre place dans les volumes marseillais et à girondin de la collection des patrons du Second Empire dirigée par Dominique Barjot. Voir Roland CATY, Éliane RICHARD et Pierre ÉCHINARD, Marseille, Paris, Picard, Le 
Mans, Éd. Cénomanes, 1999, coll. «Les patrons du Second Empire : 5 »; Hubert BonIN, Bordeaux et la Gironde, Paris, Picard, Le Mans, Éd. Cénomane, 1999, coll. « Les patrons du Second Empire : 6 ». 2. Jean Fraissinet voit le jour le 23 février 1720. Jean-Étienne Balguerie junior le 31 juillet 1756. Voir Arch. dép. Hérault, GG166, vue 47, baptême du 25/2/1720; 5 MI 1/26, vue 42, baptême du 3/8/1756.

3. Charles CARRIÈRE, Négociants marseillais au XVIII siècle: contribution à l'étude des économies maritimes, Marseille, Institut historique de Provence, 1973, 2 vol., 1111 p.; Paul BUTEL, La croissance commerciale bordelaise dans la seconde moitié du XVIII ${ }^{e}$ siècle, Service de reproduction des thèses, Université de Lille III, 1973, 2 vol.

4. Patrick VERLEY, «À propos de la "prosopographie » des élites économiques: un retour à l'anecdotique, une sociologie du patronat et/ou une nouvelle entrée dans le champ de l'histoire économique?", Revue d'histoire du $19^{e}$ siècle, 2001, n² 23, p. 202-210.

5. Claire LEMERCIER, « Analyse des réseaux sociaux », Revue d'histoire moderne et contemporaine, $\mathrm{n}^{\circ} 2$, 2005, p. 88-112.

6. Fernand BRAUDEL, Civilisation matérielle, économie et capitalisme ( $\mathrm{XV}^{e}$-XVIII ${ }^{e}$ siècle). Les jeux de l'échange, Paris, A. Colin, 1979, p. 156-157 ; Louis DERMIGNY, Cargaisons Indiennes. Solier \& Cie, Paris, S.E.V.P.E.N., 1960, coll. « Affaires et Gens d'affaires XV », Tome 1, p. 82.

7. Au milieu du siècle dernier, Louis Dermigny a consulté des papiers Balguerie conservés par une dame du même nom résidant place de la marine à Agde mais cette piste documentaire s'est malheureusement perdue. Voir G. A. CHEVALIER, G. DEBIEN, Louis DERMINY, M. GAUCHER, C. HUGUES, J. MARION, R. MASSIO et R. RICHARD, «Recherches collectives: Chronique documentaire pour une nouvelle histoire coloniale. Les papiers privés de l'Amérique française », Revue d'histoire de l'Amérique Française, vol. 6, nº 4, mars 1953. p. 541.

8. Les restitutions généalogiques disponibles en ligne ne sont pas à négliger, du moins pour une première approche car elles se révèlent toujours incomplètes et souvent erronées.

9. Archives départementales de l'Hérault [désormais Arch. dép. Hérault], C 2757, mémoire de la Chambre de commerce de Montpellier, le 8 juillet 1753.

10. La maison Laurens de Montpellier et Sète, qui figure sur la même liste, a donné lieu à un travail réalisé à partir de sa correspondance avec les Roux de Marseille. Voir Dominique SERENA, Les relations Languedoc-Marseille : la famille Estienne et Daniel Laurens. 1735-1760, mémoire de maîtrise d'histoire sous la direction de MM les professeurs Louis Dermigny et Michel Perronet, Université Paul Valéry, 1972, $101 \mathrm{p}$.

11. En 1741, il cède formellement cet enclos en partie couvert de bâtiments à sa sœur, Jeanne Fraissinet, veuve de Guillaume Sarran, qui l'utilise pour sa maison de commerce Veuve Sarran \& Bazille. Il fait retour aux Fraissinet après son décès. Voir Arch. dép. Hérault, $2^{\mathrm{E}} 60125$, vente par Antoine \& Isaac Fraissinet, 1741.

12. Arch. dép. Hérault, $2^{\mathrm{E}} 57634, \mathrm{f}^{\circ} 20 \mathrm{v}$, relation Balguerie et Desmaret, le 20 janvier $1755 ; 2^{\mathrm{E}}$ 57 640, fo 371 , quittance à Jean Balguerie, le 11 juin 1761.

13. Arch. dép. Hérault, $2^{\mathrm{E}} 90$ 23, achat par Antoine Fraissinet, le 10 mai 1754 ; 8B 406, faillite Antoine Fraissinet \& fils, 1763.

14. Arch. dép. Hérault, $2^{\mathrm{E}} 896$, $\mathrm{f}^{\circ} 118$, vente Fraissinet frères et sœurs, le 4 juillet 1767.

15. Arch. dép. Hérault, 8B 421, bilan de Marc Fraissinet \& Cie, le 7 décembre 1778.

16. Arch. dép. Hérault, 10BP 1636, 1174 (16), inventaire de Marc Fraissinet, le 27 novembre 1778.

17. Arch. dép. Hérault, 10BP 1604, 336 (2-3), inventaire de Jean Balguerie, le 28 novembre 1764.

18. Le changement de raison sociale semble être intervenu en 1738 , date d'une procuration d'Antoine à son frère Isaac. Voir Arch. dép. Hérault, étude Merle, procuration du 28 mai 1738.

19. Arch. dép. Hérault, 10 BP 1596, 225bis, Antoine Fraissinet contre Keyaert, 1750. 
20. Bien qu'associé, Marc ne reçut de son père une procuration générale qu'en février 1760 , neuf mois avant son décès. Voir Arch. dép. Hérault, $2^{\mathrm{E}} 62511$, procuration d'Antoine Fraissinet, le 22 février 1760 .

21. L'augmentation du trafic portuaire renforce progressivement l'autonomie de son activité commerciale qui fonctionne dans de nombreux cas sans le recours à des établissements montpelliérains.

22. La plupart des maisons sétoises avaient des correspondants à Bordeaux, à l'image de Rogé \& Flickwier, procureur de la maison bordelaise Karling \& Cie dans une affaire les opposant à d'autres maisons bas-languedociennes. Voir Arch. dép. Hérault, $2^{\mathrm{E}} 89$ 96, pièces annexes, procuration, le 17 octobre 1767.

23. Sur la question du rôle des négociants du Nord dans le commerce des vins bas-languedociens, voir Lionel DUMOND, «Les négociants du Nord en Bas-Languedoc jusqu'au milieu du XIX ${ }^{\mathrm{e}}$ siècle. Une approche prosopographique », Burghart SCHMIDT (dir.), Commerce et culture en Méditerranée à l'époque moderne, Hambourg, Dobu Verlag, 2010.

24. Seyffert signe au bas de l'acte de baptême de l'un des enfants de Jean Balguerie en 1753. Les baptêmes sétois de leurs enfants en témoignent. Voir Arch. dép. Hérault, GG 72, n 56, baptême de Jean-Pierre Balguerie, le 12 mai 1753.

25. Rogé poursuivait parallèlement son activité avec son gendre, le négociant hambourgeois Jean Flickwier (1719-1788). En 1762, Rogé \& Flickwier embauchent comme commis aux écritures Jurgen-Henry Eggers, un estonien de Reval né vers 1736. Voir Arch. dép. Hérault, GG17, mortuaire de Jean Flickwier, le 15 octobre $1788 ; 2^{\mathrm{E}} 89$ 17, accord Jean Flickwier et Eggers, le 31 août $1762 ; 2^{\mathrm{E}} 89$ 97, testament de Louis Rogé, le 11 mars 1765.

26. Après son décès survenu le $1^{\mathrm{e}}$ juillet 1804 , Jean-Jacques Bellaud, négociant à Montpellier et Ferdinand Seyffert neveu, négociant à Sète, intéressés dans sa maison de commerce établie sous la raison Seyffert, Bellaud \& Cie, continuent ses opérations. Voir Arch. dép. Hérault, $2^{\mathrm{E}} 8918$, pièces annexes, déclaration, le 28 juillet 1804 .

27. Dans ce cas, les firmes prélèvent un pourcentage, une commission, sur la valeur des articles expédiés.

28. Exemple de ces affaires partagées, Antoine Fraissinet \& fils a un quart du chargement d'un navire commandé en 1763 par le capitaine Dirk Oven, Veuve Pierre Deveer \& Fraissinet de Marseille, un autre quart, et Pierre Clément de Middelbourg la moitié. Voir Arch. dép. Bouchesdu-Rhône, 13 B 571, bilan de la société Veuve Pierre Deveer \& Fraissinet, négociants de Marseille, le 10 septembre 1763.

29. Pour les vins locaux, le contenant les plus usuels était le tonneau d'un demi-muid, selon une norme fixée par une ordonnance en 1718.

30. Durant le premier tiers du siècle, les exportations sétoises d'eaux-de-vie s'accrurent de $75 \%$, deux fois plus vite que celles de vins. Voir Louis DERMIGNY, Naissance et croissance d'un port. Sète de 1666 à 1880, Sète, 1955, p. 11.

31. Anne WAGENER SLEESWIJK, «La relation problématique entre principal et agent dans la commission: l'exemple de l'exportation des vins vers les Provinces-Unies au xVIII ${ }^{\mathrm{e}}$ siècle ", in Silvia MARZAGAlli et Hubert Bonin (dir.), Négoces, Ports et Océans, $\mathrm{XVI}^{e}$-XXe siècles, mélanges offerts à Paul Butel, Bordeaux, Presses Universitaires de Bordeaux, 2000, p. 31.

32. Arch. dép. Hérault, 4B 294, police de chargement, le 20 janvier 1748.

33. . Ces produits se trouvaient dans les magasins Balguerie à la fin de 1764 . Voir Arch. dép. Hérault, 10BP 1604, 336 (2-3), inventaire de Jean Balguerie, le 28 novembre 1764.

34. Exemples parmi d'autres, en avril et juillet 1734, Isaac Fraissinet faisait par exemple charger du blé pour la Ciotat et du millet pour Marseille, à l'adresse de Gaspard et Nicolas Solicoffre. Trente ans plus tard, Marc Fraissinet est de moitié sur un commerce de bois. Voir Arch. dép. Hérault, 4B 341, déclarations de chargements, les 12 avril 1734 et 6 juillet $1734 ; 2^{\mathrm{E}} 89$ 19, fo 257 , protest Marc Fraissinet, le 9 mai 1766. 
35. Cet espace commercial se superpose largement avec celui de la maison marseillaise de son frère, Veuve Pierre Deveer \& Fraissinet, failli une première fois le 10 septembre 1763 avec un passif de $218318 \mathrm{ft}$, et une seconde fois le 9 novembre 1768 avec un passif de $94630 \mathrm{Et}$. Voir Arch. dép. Bouches-du-Rhône, 13B 656, rubrique des bilans, 1723-1829.

36. Jean Balguerie \& Cie devait $90.000 \mathrm{ft}$ à Christian Cruys \& fils. Il passa un concordat le 4 mars 1763, homologué le 26. Il n'a malheureusement pas été possible de le retrouver, pas plus que le dépôt du bilan qui l'a précédé. Faut-il voir dans sa faillite l'écho des difficultés qui touchaient sa belle-famille? La question reste en suspens mais on ne peut que constater que Jean Tarteiron, marchand de draps à Montpellier sous la raison Tarteiron \& Brunet, dépose son bilan le 27 septembre 1762 et que l'entreprise Tarteiron aîné de Ganges est, à la même époque, en difficulté. Voir Arch. dép. Hérault, 8B 405, bilan Tarteiron \& Brunet, le 27 septembre 1762 et bilan André Rey \& Rech, le 16 septembre 1762.

37. En 1749, Jean Balguerie, son associé Louis Rogé et Jean Tarteiron, négociant de Montpellier, passent deux contrats de société avec Pierre Ranson \& $C^{\text {ie }}$ dans le but d'établir d'un comptoir au Cap-Français. Voir Louis DERMIGNY, Cargaisons indiennes..., op. cit., p. 82 ; Arch. dép. Hérault, 4B 284, police de chargement de la polacre Saint Esprit, 1749.

38. Lucile. BOURRACHOT, «Un inventaire de plantation à Saint-Domingue à la veille de la Révolution : la plantation Brossard-Laguehay en 1784 ", Actes du $97^{e}$ Congrés National des Sociétés Savantes, Nantes, 1972, Histoire moderne, t. II, p. 335.

39. Ces voyages s'effectuent en participation avec les marseillais Jean et David Baux. Voir François-Xavier EMMANUELLI, Les Européens et les espaces océaniques au XVIII ${ }^{e}$ siècle, Société française d'histoire d'Outre-mer, 1996, p. 82.

40. En octobre 1734, la maison Fraissinet père \& fils était l'un des six commanditaires de la construction de la tartane Sainte Rose (120 tx) à Aymes père \& fils d'Agde, dont le commandement devait être confié à Pierre Sendras pour qu'il effectue un voyage « à la caravane ». Voir Arch. dép. Hérault, 4B 341, transcription de propriété, le 28 octobre 1734 ; Gilbert BUTI, «Aller en caravane, le cabotage lointain en Méditerranée, $\mathrm{xVII}^{\mathrm{e}}$ et $\mathrm{xVIII}^{\mathrm{e}}$ siècles", Revue d'Histoire Moderne et Contemporaine, $\mathrm{n}^{\circ}$ 52-1, janvier-mars 2005, p. 7-38.

41. Leur intérêt de $20 \%$ sur l'expédition du vaisseau le Jeune Jean passe par la maison Marc Fraissinet \& fils d'Amsterdam. Voir Arch. dép. Hérault, 8B 406, faillite Antoine Fraissinet \& fils, 1763.

42. Le bâtiment de 200 tonneaux présente un devis de 20.500 £t. Voir Arch. dép. Hérault, 4B 252, devis par Antoine Cauvy, de Sète, 1771.

43. Ces six tartanes sont estimées entre 2.400 et $9.600 \mathrm{ft}$; les cinq autres bâtiments valent tous plus de $16.000 \mathrm{ft}$. Mais Marc Fraissinet ne détient généralement qu'une part minoritaire de chacun d'eux, si bien que le montant de ses participations maritimes ne s'élève qu'à $42.200 \mathrm{ft}$. Notons qu'il possède en outre une action de la frégate marseillaise Comte-de-Maupéou.

44. Cette part faisait de lui le principal propriétaire du bâtiment. En mai 1764, Balguerie donne procuration au négociant marseillais Jean-Jacques Kick pour qu'il retire en son nom le montant du fret du bâtiment de retour de Port au Prince. Voir Arch. dép. Hérault, $2^{\mathrm{E}} 89$ 17, f $5444-545$, procuration du 15 mai 1764 ; Arch. dép. Hérault, 10BP 1604336 (2-3) inventaire de Jean Balguerie, le 28 novembre 1764 .

45. En 1746, les négociants bordelais soulignaient que les expéditions bordelaises avaient été augmentées depuis la guerre par l'apport de draperies, de savons et d'huiles du Languedoc et de Provence. Voir Paul BUTEL, op. cit., tome I, p. 370.

46. Arch. dép. Hérault, $2^{\mathrm{E}} 57634$, fo $20 \mathrm{v}$, relation Balguerie et Desmaret, le 20/1/1755.

47. Gabriel-François VENEL, Instructions sur l'usage de la houille, plus connue sous le nom de charbon de terre... publiées par ordre des États... de Languedoc, Lyon, chez G. Regnault, 1775, p. 55.

48. Arch. dép. Hérault, 10BP 1604336 (2-3), inventaire de Jean Balguerie, le 28 novembre 1764. 
49. Elle permet aussi de se passer, au moins en partie des services des distillateurs installés dans les localités viticoles.

50. Antoine Fraissinet \& fils avait investi $16.000 \mathrm{ft}$ dans ces deux savonneries. Voir Arch. dép. Hérault, 8B 406, faillite Antoine Fraissinet \& fils, 1763.

51. La même modestie des biens ruraux vaut pour Jean Balguerie, acquéreur d'une maison et de vignes à Roujan, village voisin de Neffiès. Voir Arch. dép. Hérault, $2^{\mathrm{E}} 89$ 19, fo 43 , procuration par Marguerite Tarteiron, le $1^{\mathrm{e}}$ octobre 1764.

52. Arch. dép. Hérault, $2^{\mathrm{E}} 57$ 640, fo582, obligation à Marc Fraissinet, le 12 novembre 1761.

53. Concernant Balguerie, un débiteur de sa faillite avance qu'elle serait la conséquence d'un vide de $100.000 \mathrm{ft}$ dans les affaires de sa maison de commerce depuis sa séparation d'avec Rogé. Voir Arch. dép. Hérault, 10BP 1608, 422, Jean Tichy oncle contre Jean Balguerie \& Cie, faillite frauduleuse, 1763-1766.

54. Les Fraissinet lui " avaient prêté leur nom et leur crédit par des lettres de change qu'ils avaient souscrites ». À la suite de sa faillite, Bragouze s'était enfui en Suisse. Voir Henri THIRION, La vie privée des financiers au XVIII ${ }^{e}$ siècle, Paris, E. Plon, Nourrit \& Cie, 1895, p. 30 ; Peter ALLAN, Alan DAINARD, Marie-Thérèse INGUENAUD, Jean ORSONI et David SMITH (éd.), Correspondance générale d'Helvétius, vol. IV : 1774-1800 / lettres 721-855, p. 285 ; Archives municipales de Marseille, 39ii7, fonds Fraissinet, règlement de famille entre les descendants d'Antoine Fraissinet, le 21/8/1765. 55. Arch. dép. Hérault, 8B 405, bilan Lagarde père \& fils, à Béziers, le 31 juillet 1762.

56. Sa veuve cède ses actifs immobiliers sétois en 1768 . Voir Arch. dép. Hérault, $2^{\mathrm{E}} 89$ 6, f ${ }^{\circ} 338 \mathrm{v}$, vente du 14 juillet 1768 .

57. Arch. dép. Hérault, $2^{\mathrm{E}} 8910$, f ${ }^{\circ} 181$, acte de cessation de paiement, le 25 novembre 1778.

58. Il s'agit de «leur en abandonner le prix » selon les termes de son concordat signé les 18 janvier 1779 et 22 novembre 1782. La vente des immeubles doit être autorisée par le roi car Marc Fraissinet est considéré comme non catholique. Voir Arch. dép. Hérault, $2^{\mathrm{E}} 89$ 96, brevet du roi, permission de vendre, le 19 janvier 1780 .

59. Arch. dép. Hérault, $2^{\mathrm{E}} 89$ 10, f209, verbal de levée de scellées, le 15 février 1779.

60. Ce type de comportements a déjà été mis en évidence par Laurence Fontaine à partir de ses travaux sur le colportage. Voir Laurence FONTAINE, «La gestion des appartenances dans les réseaux de marchands migrants : l'Europe moderne au miroir des analyse contemporaines », in Corine MAITTE, Issiaka MANDÉ, Manuela MARTINI ET Didier TERRIER (éds); Entreprises en mouvement. Migrants, pratiques entrepreneuriales et diversités culturelles dans le monde, $\mathrm{XV}^{e}-\mathrm{XX}^{e}$ siècle, Valenciennes, Presses universitaires de Valenciennes, 2009, p. 307-321/

61. Paul BUTEL, "Marchands de Garonne entre la Méditerranée et l'Atlantique ", in Janine GARRISSON-ESTÉBE et Marc FERRo (dir.), Une histoire de la Garonne, Paris, Ramsay, 1982, p. 323-360.

62. Arch. dép. Hérault, GG 157, f $15 \mathrm{v}$, mariage d'Isaac Fraissinet et de Françoise Bousquet, le 20 avril $1691 ; 2^{\mathrm{E}} 60102$, f ${ }^{\circ} 485$, mariage de Marc Fraissinet et de François Granier, le 18 novembre $1695 ; 2^{\mathrm{E}} 57$ 386, mariage d'Adrien Fraissinet et de Françoise Despioch, le 18 avril 1699.

63. Jean Balguerie eut trois fils et quatre petits-fils qui portaient son patronyme. On compte deux Pierre parmi ces derniers. L'un, Balguerie de Paillère (1637-1717), marchand de Galapian et seigneur de Paillère, eut un fils, Pierre (1678-1759), qui s'exila à Amsterdam. L'autre, Balguerie du Metge ( $†$ 1711), du nom d'un domaine qu'il possédait à Clairac, était marchand de Clairac. Ses fils Isaac $(\dagger 1736)$ et Daniel († 1733) s'établirent négociants à Bordeaux et en Hollande.

64. L'installation d'Adrien Fraissinet à Amsterdam se situe à une date comprise entre la naissance montpelliéraine de son fils Pierre, le 29 juin 1710, et celle de son fils Jacques-Adrien survenue à Amsterdam le 23 juillet 1711. Daniel Balguerie se trouvait à Amsterdam en 1712 où il y eut un fils de Marie Delcruzel qu'il épousa en 1718 à Breukelen, à une vingtaine de kilomètres de là. Pierre Balguerie (1678-1759) épousa vers 1716 l'Amstellodamoise Théodora Rijswijk, fille d'un marchand et échevin. Il fut négociant et consul du roi de Suède de la ville. 
65. Claude MARTIN, «Réfugiés huguenots de la ville de Clairac », Bulletin de la Société de l'histoire du protestantisme français, Cahier de généalogie, $\mathrm{n}^{\circ} 39,3^{\mathrm{e}}$ trimestre 1992, p. 160.

66. Deux Clairacais arrivés à Sète vers 1725 comme tonneliers sont assez bien documentés : Louis Rogé, l'associé de Jean Balguerie, et David Cazanove (1703-1778), père de Pierre Cazanove, maître de chai de Marc Fraissinet dans les années 1770.

67. Les productions clairacaises de vins, d'eaux-de-vie et de prunes s'exportaient en Europe septentrionale en transitant par le port de la Lune. Voir Paul BUTEL, op. cit., tome I, p. 541.

68. Cette présence a probablement été assez brève. Voir Claude MARTIN, «Recherches sur la navigation de gens de rivière à Clairac auX XVII ${ }^{\mathrm{e}}$ et XVIII ${ }^{\mathrm{e}}$ siècles ", Société des sciences, lettres et arts d'Agen, Revue de l'Agenais, vol. 104, 1978, p. 28.

69. Bibliothèque municipale de Bordeaux, $\mathrm{ms}$ 1868/12, lettre de Pierre Balguerie à Charles-Louis Secondat datée d'Amsterdam, le 9 octobre 1749 ; Paul BUTEL, op. cit., tome I, p. 456.

70. Une expédition d'Isaac Fraissinet pour Amsterdam est répertoriée en 1720 tandis que sa présence à Sète est attestée trois ans plus tard par la naissance de sa fille Thérèse-Marguerite dans le port. Voir Arch. dép. Hérault, CG 6, f०42, baptême du 19 avril 1723.

71. Jean-Claude GAUSSEN, « Un aspect du commerce maritime sétois au XVIII ${ }^{\mathrm{e}}$ siècle : l'exportation des vins et eaux-de-vie par les bâtiments du Nord", Bulletin du Centre d'histoire des Espaces Atlantiques, $\mathrm{n}^{\circ} 3,1987$, p. 99.

72. Marchand amstellodamois en 1730, Marc est qualifié de négociant trois ans plus tard. Voir Arch. dép. Hérault, $2^{\mathrm{E}} 60$ 117, fo 354 vº , versement d'Isaac Fraissinet, le 26 décembre 1730 ; GG170, baptême de Constance-Marie Fraissinet, le 5 décembre 1733.

73. Le concordat passé par Veuve Pierre Deveer \& Fraissinet s'était traduit par une remise des trois quarts de leurs créances. Marc se chargea de celles envers la manufacture de savons établie à Sète sous la raison Joseph Degroux \& Cie. Voir Arch. dép. Hérault, $2^{\mathrm{E}} 89$ 6, fo $278 \mathrm{v}$, quittance Marc Fraissinet, le 27 avril 1768.

74. Arch. dép. Bouches-du-Rhône, registre de non catholiques, 1788 , fo 18 , rectification du mariage de Marc Fraissinet, le 29 mars 1788 ; Arch. dép. Hérault, $3 Q$ 10140, fo 46 , déclaration de succession de Marc Fraissinet, le 23 frimaire an $\mathrm{x}$.

75. La présence de ce Fraissinet, fils de Fraissinet de Montpellier, est attestée en 1791. Il y serait arrivé par le Constance cette même année pour remplacer Vialars. Mais il y est probablement depuis la création de la maison Vialars \& Cie en 1785. Il s'agit probablement de Pierre-Jean-Marc, fils de Marc, qui s'établit plus tard courtier à Marseille. Voir Louis DERMIGNY, Cargaisons indiennes, op. cit., p. 130 et 165.

76. L'inventaire de ses biens comprend en effet des papiers et comptes antérieurs à 1745 relatif à une affaire située à Montréal. Voir Arch. dép. Hérault, 10BP 1604, 336 (2-3), inventaire de Jean Balguerie, le 28 novembre 1764.

77. André Rogé $(† 1775)$, frère de Louis ( $† 1765)$ voit en effet le jour à Bordeaux vers 1701 . Voir Arch. dép. Hérault, GG 17, inhumation d'André Rogé, le 20 décembre 1775.

78. Son nom se trouve orthographié avec diverses terminaisons dans les sources: il est écrit Verborg, Verborch ou encore Verboroch. Rogé et Verborg expédient pour Rotterdam dès 1731. La dernière mention relevée d'une de leurs expéditions est datée de 1743 . Voir Jean-Claude GAUSSEN, «Un aspect du commerce maritime sétois », art. cit., p. 101 et 106 ; Guillaume GERAUD-PERRACHA, Le commerce des vins et des eaux-de-vie en Languedoc sous l'Ancien régime, thèse de droit, Montpellier, 1955, p. 183.

79. Antoine Verborch réside à Hambourg en 1771 et s'y qualifie de «ci-devant négociant de Montpellier». Voir Arch. dép. Hérault., $2^{\mathrm{E}} 89$ 96, pièces annexes, procuration devant JeanChristophe Verst, notaire à Hambourg, à Louis Rogé, le 6 juillet 1771.

80. Illustrations de cette fortune, Jean Balguerie consentait divers prêts en 1751 ; il recevait $10.000 \mathrm{ft}$ en 1759 pour le reste du prix d'une maison située à Bordeaux qu'il avait cédé à son 
oncle, le négociant bordelais Dominique Rion. Voir Arch. dép. Hérault, $2^{\mathrm{E}} 57630$, f ${ }^{\circ} 256$, obligation à Balguerie du 24 mai $1751 ; 2^{\mathrm{E}} 57$ 638, fo 425 , dépôt de brevet par Jean Balguerie, le 3 septembre 1759.

81. Arch. dép. Hérault, 4B 293, amirauté de Sète, déclarations des 13 juin et 24 décembre 1747.

82. Jean-Claude GAUSSEN, « Un aspect du commerce maritime sétois », art. cit., p. 106.

83. Le premier enfant de Jean Balguerie et de Marguerite Tarteiron nait à Montpellier en mars 1749. À cette date, Jean est absent de la ville, mais son jeune frère Dominique le représente auprès de son épouse. Voir Arch. dép. Hérault, (par saint Denis) ; f 373 , baptême de Jeanne Balguerie, le 27 mars 1749 .

84. En 1758, à Bordeaux, Dominique Balguerie est le parrain de son neveu Louis-Alexandre Demontis. Voir Perre MELLER, Les familles protestantes de Bordeaux, Bordeaux, E. Crugy, 1902, p. 43.

85. En 1783, ces expéditions représentent $883.000 \mathrm{lb}$ de sucre et $723.000 \mathrm{lb}$ de café. Voir Paul BUTEL, op. cit., tome I, p. 351 ; Jean SAGNES, Agde sous la Révolution, mémoire de DES sous la direction de M. le Professeur Laurent, Faculté des Lettres, Université de Montpellier, 1961, p. 39.

86. C'est le cas au début de la Révolution lorsqu'ils furent chargés de l'expédition de 1.500 boisseaux de blé à destination du Lot-et-Garonne. Geste patriotique ou humanitaire, ils refusèrent toute rétribution pour leurs frais d'expédition.

87. Paul BUtel, op. cit., tome I, p. 369, 419 et 421.

88. Reçu capitaine au long cours, Balguerie Junior commande dès 1779, à l'âge de 23 ans, le Darcy pour un voyage à Saint-Domingue. Il entreprend quatre ans plus tard un voyage de circumnavigation qui fait de lui le troisième français à réaliser un tout du monde à la tête d'un navire. Voir Silvia MARZAGALLI et Hubert BONIN, op. cit., p. 105.

89. Philippe GARDEY, Négociants et marchands de Bordeaux de la guerre d'Amérique à la Restauration (1780-1830), Paris, PUPS, 2009, p. 729.

90. Silvia marzagali, Les boulevards de la Fraude: le négoce maritime et le Blocus continental, Lille, Septentrion, 2000, p. 288.

91. Patrick VILLIERS, " Navires corsaires et constructions navales de Louis XIV à Napoléon $\mathrm{I}^{\mathrm{e}}$ ", in Jean-Pierre Poussou, Revue d'Histoire Maritime $n^{\circ}$ 7. Les constructions navales dans l'histoire, p. 73.

92. Le bâtiment de 169 tonneaux fait naufrage en 1818 sur les côtes d'Espagne. Armé à Marseille pour Nantes, il était sous le commandement de Jacques-Léonce Martin, de Saint-Tropez.

93. . Ces caractères sont présentés par Éliane Richard comme étant à la source de la réussite sociale et professionnelle des Fraissinet de Marseille au siècle suivant. Voir Éliane RICHARD, « Un siècle d'alliance et d'ascension sociale : Les Fraissinet ", Provence historique, 142 (1985), p. 423.

94. Bien entendu, les Balguerie et les Fraissinet s'inscrivent aussi dans des réseaux dont la parenté n'est pas le critère central d'appartenance : ceux, notamment des églises réformées, des institutions éducatives et des loges maçonniques. On regrettera particulièrement de n'avoir pas exploité cette dernière piste prometteuse du côté des Balguerie, car leurs liens avec Marseille passent par les Tarteiron et les Hugues, deux familles influentes de la loge à dominante négociante et protestante qu'est Saint-Jean d'Écosse, et parce que des loges correspondantes de Saint-Jean d'Écosse se trouvent, à Montpellier, Sète ou Malte, inscrivent dans l'espace de leur réseau. Voir Pierre-Yves BEAUREPAIRE, «Saint-Jean d'Écosse de Marseille, une puissance maçonnique méditerranéenne aux ambitions européennes », Cahiers de la Méditerranée, $\mathrm{n}^{\circ} 72$, juin 2006, p. 73 et 85 .

95. Arch. dép. Bouches-du-Rhône, registres paroissiaux, paroisse Saint-Martin, le 14 juillet 1748, $\mathrm{f}$ ${ }^{\circ} 125, \mathrm{n}^{\circ} 148$, mariage de Jean Balguerie et de Marguerite Tarteiron ; 363 ${ }^{\mathrm{E}} 316$, contrat du mariage de Jean Balguerie, 17 juillet 1748.

96. Originaire de Lagrand (Hautes-Alpes), Antoine Hugues forme avec ses frères Joseph, Gaspard et Jacques une fratrie de grands négociants, membres du patriciat marseillais, intéressés au 
commerce des Antilles. Son frère Joseph passe pour le plus riche négociant et armateur de Marseille à la veille de la Révolution. Voir Louis DERMIGNY, Cargaisons indienne, op. cit., p. 257.

97. Négociants à Marseille, les frères Isnard sont, avec Deveer \& Fraissinet, parmi les clients de la maison Bordelaise Balthazard David \& Cie au début des années 1760. Ils s'illustrent notamment par leur activité maçonnique dans la loge Saint-Jean d'Ecosse de Marseille, et on les retrouve plus tard dans la maison de commerce Isnard-Audibert conduite par Dominique Audibert (1736-1821). Voir Arch. dép. Gironde, 7B 1255, archives Balthazard David \& Cie. Voir Pierre-Yves BEAUREPAIRE, art. cit., p. 72.

98. Isaac Tarteiron (ca 1680-1768) s'établit à Marseille au début du siècle et y épouse en 1717 Marguerite Murat, fille de François Murat et de Marguerite Tandon. Fils et petit-fils de marchands-tanneurs gangeois, son grand-père Isaac Tarteiron est le frère du marchand gangeois Guillaume Tarteiron, l'arrière-grand-père de Marguerite Tarteiron (1722), fille de Laurent Tarteiron (ca 1787), marchand-fabricant de bas à Ganges, et petite-fille de Pierre Tarteiron, marchand-apothicaire.

99. Ce groupe forme avec les Seymandi et les Audibert un ensemble de familles associées en affaires et alliées par de multiples liens matrimoniaux, qui se retrouve à la Chambre de commerce de la ville et, plus tard, au sein de la loge marseillaise Saint-Jean d'Écosse. Certains Audibert de Marseille étaient d'extraction clairacaise au moment de la Régence. Voir Claude MARTIN, « Recherche sur la navigation », art. cit., p. 28.

100. Jean Tarteiron (1694) est alors négociant de Montpellier. Oncle de Marguerite Tarteiron, l'épouse de Jean Balguerie, il s'est marié en 1723 à Marie-Catherine Lajard (1704-1744), ce qui le rattache aux Lajard, grande famille du négoce protestant montpelliérain. L'entreprise de SaintDomingue associe sa firme Tarteiron \& Cie à Rogé \& Balguerie et à des commanditaires genevois, Roque \& Bonnafous. Voir Arch. dép. Hérault, $2^{\mathrm{E}} 57635, \mathrm{f}^{\circ} 484$, reconnaissance par Jean Balguerie à Laurent Tarteiron, le 20 septembre 1756.

101. Jean Amphoux, marchand de Marseille, offre $8.500 \mathrm{ft}$ pour l'achat de sa maison saisie dans le cadre de sa procédure comme fugitif. Voir arch. dép. Hérault, 1B 40, procédure de saisie des biens d'Adrien Fraissinet, le 27 septembre 1714.

102. En 1763, ces liens concernent les maisons Antoine Fraissinet \& fils de Montpellier et Deveer Bugnot \& Cie d'Amsterdam, et, Marc Fraissinet \& fils d'Amsterdam. Voir Jean-Claude GAUSSEN, Les protestants et l'église réformée de Sète, thèse sous la direction de Michel Perronet, Université Paul Valéry, 1992, p. 668 ; Arch. dép. Bouches-du-Rhône, 13 B 571, bilan de la société Veuve Pierre Deveer \& Fraissinet, négociants de Marseille, le 10 septembre 1763.

103. Arch. dép. Bouches-du-Rhône, registres paroissiaux, paroisse Saint-Martin, le 7 mai 1749, $\mathrm{f}^{\circ}$ 86, mariage de Jean Fraissinet et de Marguerite Fraissinet.

104. Il déclare avoir «le mariage pour agréable». Voir Arch. mun. Marseille, 39ii7, fonds Fraissinet, contrat de mariage passé devant Me Courmes, le 16 juillet 1750.

105. En 1757, Jean Fraissinet séjourne à Livourne pour la société Veuve Deveer \& Fraissinet, nouveau nom de son entreprise après le décès de Pierre Deveer en 1754 et le retrait d' Henri Deveer.

106. En 1769, Joseph Arnaud est présenté comme négociant bordelais lors du baptême d'un des enfants de Marc. Paul Butel le désigne comme commissionnaire en farines en 1776. Voir Paul BUTEL, op. cit., tome I, p. 478.

107. Il existe bien un Jacques Fraissinet établi à Bordeaux, armateur et négociant dans le port de la Lune au début des années 1750, mais il n'a apparemment pas de rapports avec un autre Jacques Fraissinet (1708), fils d'Adrien Fraissinet établi à Amsterdam, qui lui relève de la famille qui nous intéresse ici. De même, les Fraissinet établis à Toulouse et à Montauban dans les années 1730-1740 n'ont pas de liens avec les Fraissinet de Montpellier.

108. Les intérêts des frères Périer s'étendent de Montpellier à Sète et de Sète à Marseille. Antoine Seimandy est le cousin-germain du négociant marseillais Jacques Seimandy. 
109. Cette pratique ne va pas sans poser de problème lorsque Marc doit rembourser les sommes restant dues sur les dots de sa cousine et de sa tante Anne Gervais, mais il parvient à conclure un accord familial en 1765.

110. Arch. dép. Hérault, $2^{\mathrm{E}} 57$ 632, fº 497 , mariage de Marc Fraissinet, le 19 novembre 1753.

111. La veuve Sarran, née Fraissinet, est une sœur d'Antoine Fraissinet.

112. Étienne Demontis, d'abord établi à Cognac, a probablement profité de son mariage pour s'installer à Bordeaux.

113. On peut également citer le choix de Marsac, sur la commune de Clairac, pour le mariage au désert de Jeanne Balguerie (1749-1827), fille aîné de Jean, en 1765, avec Jean-Louis dit Alexis Baour (1750-1830).

114. . Son fils P. Boué figure en 1753 parmi les principaux commissionnaires hambourgeois pour l'importation du sucre. Klaus WEBER, «Les livres douaniers de l'amirauté de Hambourg au XVIIIe siècle, une source de grande valeur encore inexploitée ", Bulletin du centre d'histoire des espaces atlantiques, 1999, p. 101.

115. S'il est de tradition de faire parrainer les premiers enfants par leurs grands-parents, le choix devient ensuite d'autant plus large que le nombre d'enfants est élevé.

116. Ce baptême, qui est l'un des seuls à se dérouler à Sète, voit signer nombre de négociants sétois de premier plan dont Bresson et Garonne. Voir Arch. dép. Hérault, GG19, vue 72, baptême du 29 mars 1762.

117. Ce Jean Tarteiron (c.a. 1734-1822) est le fils du marchand-tanneur gangeois Isaac Tarteiron, lui-même cousin-germain d'Isaac Tarteiron de Marseille dont il a été question plus haut.

118. Le mariage est célébré à Montpellier, lieu de résidence de la mère de l'épouse, en présence de Jean-Louis Baour. Jean-Louis Baux y est dit citoyen de Genève résident à Bordeaux, fils de Jean-Louis Baux, bourgeois de Genève et de Judit Tardieu. Voir Arch. dép. Hérault, 5 Mi 1/69 vue 18, mariage du 28 juillet 1778.

119. Pierre MELLER, op.cit. p. 50.

120. Louis DERMIGNY, Cargaisons indiennes, op. cit., p. 186.

121. Outre Marseille, Jean Tarteiron serait aussi passé par Lyon et par Genève. Il est négociant à Bordeaux lorsqu'il y épouse en 1768 Jeanne Borel, la fille du négociant Pierre Borel. Il se remarie en 1787 avec Sophie-Victoire Dupuy de Montbrun. Son frère aîné, Isaac Tarteiron (1728-1813), dit Tarteiron de Saint-Rémy, s'est fixé à Bordeaux en 1765 après avoir fait plusieurs voyages à la Martinique depuis Marseille. En 1756, il a épousé à Bouliac Madeleine Boucherie, la fille du négociant bordelais Antoine Boucherie. Les deux frères Tarteiron deviennent de grands négociants et armateurs bordelais. Voir Louis DERMIGNY, Cargaisons indienne, op. cit., p. 186 ; Philippe GARDEY, op. cit., p. 248 ; Pierre MELLER, op. cit., p. 56.

122. Il est le fils du négociant bordelais Pierre Duret de la Plane et de Gertrude Winthuysen (1728-1778), qui semble être la fille du négociant bas-languedocien originaire des ProvincesUnies Winthuysen établi à Frontignan et à Sète.

123. Les mêmes comportements se retrouvent du côté des enfants de Jean Fraissinet de Marseille: le négociant amstellodamois Jacques-Marc Fraissinet van Arp, cousin de Jean Fraissinet, est le parrain de son fils Jacques-Marc puis son gendre après lui avoir donné en mariage sa fille Henriette-Élisabeth.

124. En 1749 et 1754, Jeanne Balguerie et Jean-Paul Balguerie ont chacun une marraine bordelaise, respectivement leur grand-mère et leur tante paternelle.

125. Jean-Etienne Balguerie, dit Balguerie junior, commande l'année suivante le Farci pour SaintDomingue.

126. Magdeleine Méry se présente comme la fille de Pierre Méry, victime des « dissensions civiles qui agitaient la France " sous la Révolution et de la caennaise Jeanne Carruë (1761-1826). Voir Arch. dép. Hérault, $3^{\mathrm{E}} 154 / 23$, fo 24 , mariage du $1^{\mathrm{e}}$ avril 1837. 
127. D'autres indicateurs permettraient de parvenir aux mêmes conclusions, et notamment les parcours des nombreux négociants nordiques venus s'installer à Sète du XVIII ${ }^{\mathrm{e}}$ siècle au milieu du siècle suivant qui étaient généralement passés par Bordeaux ou, avec une moindre régularité, par Marseille.

\section{AUTEUR}

\section{LIONEL DUMOND}

Maître de conférences en histoire contemporaine, Centre de Recherches Interdisciplinaires en Sciences humaines Et Sociales (CRISES, EA 4424), Université Paul Valéry - Montpellier III, Site Saint Charles, Rue du Professeur Henri Serre, 34090 Montpellier 